\title{
Target Interaction of Cas9-sgRNA Influences DNA Double Strand Break Repair Pathway Choices in CRISPR/Cas9 Genome Editing
}

\section{An-Yong Xie ( $\sim$ anyongxie@zju.edu.cn )}

Zhejiang University https://orcid.org/0000-0002-6608-2550

Si-Cheng Liu

Zhejiang University

Yi-Li Feng

Zhejiang University https://orcid.org/0000-0002-7143-9956

Xiu-Na Sun

Zhejiang University

Ruo-Dan Chen

Zhejiang University

Qian Liu

Zhejiang University

Jing-Jing Xiao

Zhejiang University

Ji-Feng Xiang

Zhejiang University

Guo-Qiao Chen

Zhejiang University

Yi Yang

Zhejiang University

Chao Lou

Hangzhou Shu Rui Tech

Hao-Dan Li

Hangzhou Shu Rui Tech

Shi-Ming Xu

Zhejiang University

Hui Lin

Zhejiang University 
Keywords: CRISPR-Cas9 genome editing, DNA double strand break repair pathway choice, editing heterogeneity, off-target effect, target interaction

Posted Date: February 14th, 2022

DOl: https://doi.org/10.21203/rs.3.rs-764726/v1

License: (c) (1) This work is licensed under a Creative Commons Attribution 4.0 International License. Read Full License 


\section{Abstract}

Background Due to post-cleavage residence of the Cas9-sgRNA complex at its target, Cas9-induced DNA double strand breaks (DSBs) have to be exposed in order to engage DSB repair pathways. Target interaction of Cas9-sgRNA determines its target-binding affinity and modulates its post-cleavage targetresidence duration and exposure of Cas9-induced DSBs. This exposure by different mechanisms may initiate variable cellular DNA damage response, thus influencing DSB repair pathway choices and contributing to mutational heterogeneity in genome editing. However, this regulation of DSB repair pathway choices is poorly understood.

Results In repair of Cas9-induced DSBs, repair pathway choices vary widely at different target sites and classical non-homologous end joining (c-NHEJ) is not even engaged at some sites. Weakening target interaction of Cas9-sgRNA promotes bias towards c-NHEJ, which is intrinsically accurate for Cas9induced DSBs. As an important strategy for enhancing homology-directed repair, inactivation of c-NHEJ however aggravates off-target activities of Cas9-sgRNA due to its weak interaction with off-target sites. By dislodging Cas9-sgRNA from its cleaved targets, DNA replication alters DSB end configurations and suppresses c-NHEJ in favor of other repair pathways whereas transcription has little effect on DSB repair pathway choices. Dissociation of Cas9-sgRNA from its cleaved target by DNA replication may generate three-ended DSBs, resulting in palindromic fusion of sister chromatids, a potential source for CRISPR/Cas9-induced on-target chromosomal rearrangements.

Conclusions Target interaction of Cas9-sgRNA modulates DSB repair pathway choices likely through varying dissociation of Cas9-sgRNA from cleaved DNA, thus widening on-target and off-target mutational spectra in CRISPR/Cas9 genome editing.

\section{Highlights Of Study}

- Novel series of 1, 2, 3-triazole tethered pyridine hybrid derivatives were synthesized.

- All the synthesized compounds were screened in vitro against cytotoxicity against two human cancer cell lines like HT-1080 (Human fibro sarcoma cells) and Caco-2 (Human colorectal adenocarcinoma cells).

- $\mathrm{IC}_{50}$ values were compared against standard anti cancer drug doxorubicin.

- Among the series, compounds $7 \mathrm{~b}, 7 \mathrm{c}$ and $\mathbf{7 f}$ have showed in-vitro cytotoxicity.

- In silico molecular docking studies of these compounds $7 \mathrm{~b}$ and $7 \mathrm{f}$ shows maximum dock scores.

\section{Introduction}

CRISPR/Cas9 genome editing relies on the binding of the Cas9 nuclease, in complex with a single guide RNA (sgRNA), to a DNA target to induce a site-specific DNA double strand break (DSB) and its subsequent repair $[1,2]$. Upon DSB induction by Cas9, different repair pathways compete for DSB repair, generating the desired DNA edits including substitutions, insertions, deletions or translocations among varieties of 
repair products [3]. The two major DSB repair mechanisms in mammalian cells include non-homologous end joining (NHEJ) and homology-directed repair (HDR). While classical NHEJ (c-NHEJ) is the primary NHEJ pathway, alternative NHEJ (a-NHEJ) could also be employed to re-ligate the ends of DSBs if either of the core NHEJ factors including DNA-PKcs, Ku70/Ku80, XRCC4/DNA ligase 4 is deficient or not engaged [4]. If the ends of DSBs are readily ligatable, such as Cas9-induced blunt ends and I-Scel-induced 3 '-overhanging ends, c-NHEJ generates largely accurate end-joining products whereas a-NHEJ remains mostly mutagenic $[5,6]$. Additionally, using homologous sequences as a template, HDR is the preferred pathway for accurate substitutions and insertions in CRISPR/Cas9 genome editing.

The DSB repair pathway choice is governed by a host of factors, including cell cycle stage, DNA end configurations, surrounding chromatin context and local DNA metabolism [7]. Uniqueness in DSB induction by CRISPR/Cas9 may also participate in this regulation [8,9]. In CRISPR/Cas9 genome editing, targeting Cas 9 to a given site is mediated by several interactions, including the contacts between Cas 9 and the protospacer adjacent motif (PAM) of the target, the base pairing of the sgRNA spacer with target strand and non-specific interactions between Cas9 and target DNA [2]. In vitro and in vivo studies have indicated that these interactions entail strong and persistent binding of the Cas9-sgRNA complex to its target and help maintain its target residence for hours (h) even after Cas9-induced DNA cleavage [10-14]. Repair kinetics reveals that repair of Cas9-induced DSBs is generally slow and often lasts for more than $20 \mathrm{~h}$ in mammalian cells; this is likely due to the concealing of DSBs by the Cas9-sgRNA complex retained at the cleaved DNA $[14,15]$. Owing to intrinsic disparity in the interactions that mediate the binding of Cas9-sgRNA to its target, the binding affinity of Cas9-sgRNA varies at different sites along with altered target residence. It is likely that Cas9-sgRNA could be spontaneously released from its target, or may encounter local DNA replication, transcription or chromatin remodeling, leading to release of Cas9sgRNA from cleaved DNA and exposure of Cas9-induced DSBs $[8,9,16-19]$. These DSBs are subsequently recognized and engaged with repair factors that determine a pathway choice. Therefore, Cas9-sgRNA target interaction may regulate DSB repair pathway choices in CRISPR/Cas9 genome editing, as this interaction can persist even after DNA cleavage. However, this hypothesis has yet to be tested. Even if target interaction of Cas9-sgRNA affects repair of Cas9-induced DSBs, it is unclear what effect it has on repair of Cas9-induced DSBs and how.

Here, we find that the extent of C-NHEJ involvement varies between different target sites in repair of Cas9induced DSBs in a population of asynchronous mammalian cells. We demonstrate that weakening target interaction of Cas9-sgRNA promotes the repair bias toward c-NHEJ at the same Cas9-induced DSBs. The c-NHEJ inhibition, which is often used to increase HDR-mediated CRISPR/Cas 9 genome editing, elevates off-target effects of CRISPR/Cas9, as the interaction between Cas9-sgRNA and off-target sites is weaker. Local DNA replication, not transcription, suppresses c-NHEJ and promote a-NHEJ and HDR by dislodging Cas9-sgRNA that remains bound to its cleaved target and generating three-ended DSBs unsuitable for CNHEJ. Repair of three-ended DSBs could result in palindromic fusion of sister chromatids, a key step in chromosomal breakage-fusion-bridge cycles and a potential source for on-target gross chromosomal rearrangements in CRISPR/Cas9 genome editing. As CRISPR/Cas9 genome editing generates highly heterogeneous repair products, the effects of Cas9-sgRNA target interaction on DSB repair pathway 
choices at both on-target sites and off-target sites may significantly contribute to this mutational heterogeneity.

\section{Results}

\section{Inactivation of c-NHEJ induces varying stimulation of Cas9-induced HDR among targets}

Like any other DSBs, Cas9-induced DSBs are repaired by c-NHEJ, a-NHEJ and HDR (Fig 1a). Thus, inactivation of the predominant NHEJ pathway c-NHEJ is expected to channel more Cas9-induced DSBs towards HDR for repair, increasing the usage of HDR [20-22] (Fig 1a). If target interaction of Cas9-sgRNA influences DSB repair pathway choice after DNA cleavage at its targets, the involvement of c-NHEJ in repair of Cas9-induced DSBs would change between targets with different target interaction for Cas9sgRNA. Inactivation of c-NHEJ would thus lead to varying degrees of HDR stimulation at these sites. To test this hypothesis, we used Streptococcus pyogenes Cas9 (SpCas9) in complex with its sgRNA partner (Cas9-sgRNA) to induce site-specific DSBs at different sites in a single-copy HDR reporter integrated at the Rosa26 locus in the genome of mouse embryonic stem cells (mESC) and analyzed the impact of cNHEJ inactivation on Cas9-induced HDR (Fig 1a). This HDR reporter contains two inactivated GFP copies, TrGFP truncated at the 5 '-end and I-Scel-GFP interrupted with an 18-bp recognition site for the rare cutting endonuclease I-Scel [23]. Using TrGFP of the sister chromatid as a template, HDR of a site-specific chromosomal DSB induced by I-Scel or CRISPR nucleases generates a wild-type GFP copy and thereby $G F P^{+}$cells (Fig 1a). The frequency of $G F P^{+}$cells induced by I-Scel or CRISPR nucleases reflects the level of HDR. Like I-Scel-induced HDR, Cas9-induced HDR was increased by NU7441 at the sites targeted by $\mathrm{gHR}_{\mathrm{C}} 1, \mathrm{gHR}_{\mathrm{C}} 2$ and $\mathrm{gHR}_{\mathrm{C}} 3$ and the extent of this stimulation was different among these three targets (Fig 1b). Surprisingly, DNA-PKcs inhibition did not elevate HDR induced by Cas9-gHR 4 and Cas9-gHR 5 (Fig 1b), suggesting a possibility of little c-NHEJ involvement in DSB repair at either the gHR 4 site or the $\mathrm{gHR}_{\mathrm{C}} 5$ site. We also used CRISPR/Cas9 gene editing to generate isogenic wild-type, DNA-PKCS ${ }^{-/}$and $\mathrm{Ku} 8 \mathrm{O}^{-/-} \mathrm{mESC}$ clones containing the HDR reporter (Additional file 1, Fig S1a, b). Using one of these clones, along with isogenic $X R C C 4^{+/+}$and $X R C C 4^{-/-}$HDR reporter mESC previously established [24], we found that deletion of DNA-PKCS, Ku80 orXRCC4 significantly enhanced HDR induced by $\mathrm{gHR}_{\mathrm{C}} 1, \mathrm{gHR}_{\mathrm{C}} 2$ or $\mathrm{gHR}_{\mathrm{C}} 3$ in complex with SpCas9, as well as HDR induced by I-Scel (Fig 1c and Additional file 1, Fig S1c). However, deletion of DNA-PKCS or Ku80 stimulated no HDR at the $\mathrm{gHR}_{C} 4$ and $\mathrm{gHR}_{\mathrm{C}} 5$ sites whereas deletion of XRCC4 caused limited degrees of HDR stimulation at these two sites (Fig 1c). Therefore, the extents of HDR stimulation by c-NHEJ inactivation varied among these five different targets from little stimulation at the $\mathrm{gHR}_{\mathrm{C}} 4$ and $\mathrm{gHR}_{\mathrm{C}} 5$ sites to stimulation by $90.7 \%$ at the $\mathrm{gHR}_{\mathrm{C}} 2$ target (Fig $1 \mathrm{C}$ ). It is possible that c-NHEJ is engaged to different extents among targets where Cas9-induced HDR is stimulated to varying degrees by inactivation of c-NHEJ, and not even engaged at all at the targets where Cas9-induced HDR is not stimulated by inactivation of c-NHEJ.

\section{Repair of Cas9-induced DSBs involves c-NHEJ to varying degrees at different targets}


To directly analyze the extent of c-NHEJ involvement in repair of Cas9-induced DSBs at different target sites, we used Cas9-sgRNA to induce site-specific DSBs in an NHEJ reporter integrated in the genome of mESC as done before [25] and analyzed the effect of c-NHEJ inactivation on the frequencies of Cas9induced insertion or deletion mutations (indels) (Fig 1d). In this NHEJ reporter, no wild-type GFP is translated due to an upstream, out-of-frame translation start site (Koz-ATG), which is flanked by two I-Scel sites sequentially positioned [26]. When a DSB is induced by Cas9-sgRNA at a site between "Koz-ATG" and the ATG-GFP coding region, repair by either c-NHEJ or a-NHEJ can generate indels at the repair junction. In theory, only a third of indels can lead to $G F P^{+}$cells, and the frequency of Cas9-induced $G F P^{+}$ cells thus represents the relative efficiency of Cas9-induced indels [25] (Fig 1d). As c-NHEJ and a-NHEJ generate different proportions of accurate NHEJ (accNHEJ) products and indel-based mutagenic NHEJ (mutNHEJ) products [4], inactivation of c-NHEJ would channel more Cas9-induced DSBs towards errorprone a-NHEJ in addition to HDR, altering the frequencies of mutNHEJ. We found that neither DNA-PKcs inhibition by NU7441 nor XRCC4 deletion changed the frequencies of mutNHEJ represented by Cas9induced $\mathrm{GFP}^{+}$cells at the two sites targeted by the sgRNA $\mathrm{gEJ}_{\mathrm{W}} 3$ or $\mathrm{gEJ}_{\mathrm{W}} 7$, suggesting little involvement of c-NHEJ at these two sites (Fig 1e). However, inactivation of c-NHEJ inhibited the level of Cas9-induced $G F P^{+}$cells at the four sites targeted by $\mathrm{gEJ}_{\mathrm{C}} 5, \mathrm{gEJ}_{\mathrm{W}} 4, \mathrm{gEJ} \mathrm{J}_{\mathrm{W}} 5$ and $\mathrm{gEJ} \mathrm{J}_{\mathrm{W}} 6$ to different extents, varying from $16.6 \%$ to $69.2 \%$ (Fig 1 e). This indicates that the participation of c-NHEJ varies in repair of Cas9-induced DSBs at different targets.

Additionally, using targeted PCR amplicon deep sequencing as done before [25], we measured the frequencies of Cas9-induced indels at two natural genome loci Cola 1 and Rosa26 in mESC. We found that NU7441 reduced the editing efficiency at the sites targeted by Cola $1 \mathrm{gC} 2$ and Rosa26 gR3, stimulated by more than 2 -fold at the sites by Cola $1 \mathrm{gC} 3$, and had minimal effect at the rest of the sites including gC1 and gC4 for Cola1 and gR1, gR2 and gR4 for Rosa26 (Fig 1f, g). Together with varying stimulation of Cas9-induced HDR at different targets by inactivation of c-NHEJ, these results suggested variable involvement of c-NHEJ in CRISPR/Cas9 genome editing at different sites or even no involvement of cNHEJ at some sites.

\section{Target recleavage by Cas 9 amplifies the mutagenicity of c-NHEJ}

Like I-Scel, CRISPR nucleases generate DSBs with directly ligatable ends. Previous studies have demonstrated that c-NHEJ is intrinsically accurate for these ends $[5,6,26]$. In each round of repair during CRISPR/Cas9 genome editing, about a half of NHEJ products are accurate in repair of Cas9-induced DSBs and the remaining half generate indels [5]. Thus, inactivation of c-NHEJ would increase the use of a-NHEJ in each round of CRISPR/Cas 9 genome editing. Since a-NHEJ is more error-prone, inactivation of c-NHEJ would elevate Cas9-induced indels. It is unexpected that the frequency of Cas9-induced indels was instead inhibited at many Cas9-sgRNA target sites by inactivation of c-NHEJ (Fig 1e-g). To determine whether this was unique to repair of Cas9-induced DSBs, we used the same NHEJ reporter cells but with the first I-Scel site being deleted to ensure that I-Scel induces single cleavage as Cas9 does and compared the effect of c-NHEJ inactivation on the frequency of Cas9- and I-Scel-induced indels represented by GFP ${ }^{+}$ 
cells (Additional file, Fig S2a). In consistent with previous findings that inactivation of c-NHEJ stimulates production of I-Scel-induced $G F P^{+}$cells [25,27], inhibition of c-NHEJ with NU7441 increases I-Scel-induced $\mathrm{GFP}^{+}$cells by more than 2-fold (Additional file 1, Fig S2b, c). Given the fact that inactivation of c-NHEJ suppresses Cas9-induced indels at many Cas9-sgRNA target sites, this appears to suggest a difference between Cas9- and I-Scel-NHEJ.

We then wondered what the difference is. While c-NHEJ of both I-Scel- and Cas9-induced DSBs generates a significant level of accurate end-joining products in each round of repair at their respective targets, regenerating the target sites for recleavage, the recelavage by Cas 9 may be much more efficient than IScel $[28,29]$. Thus, in cells expressing abundant Cas9-sgRNA, these target sites could be efficiently recleaved and repaired until indels are introduced and accumulated (Fig 2a). As a result, c-NHEJ appeared mostly mutagenic for Cas9-induced DSBs and inactivation of c-NHEJ would reduce Cas9-induced indels (Fig 1e). To test this possibility, we reduced the transfection amount of Cas 9 or sgRNA into the NHEJ reporter $\mathrm{mESC}$ to limit the Cas9 recleavage in the cells and determined whether Cas9-induced $G F P^{+}$cells would be stimulated by DNA-PKcs inhibition after Cas9 recleavage is restricted (Fig 2a). We found that overall Cas9-induced $G F P^{+}$cells was reduced with a low amount of Cas9-gEJ $J_{W} 6$ in the absence of cNHEJ inhibition (Fig 2b). This could be explained by either less initial Cas9 cutting, less Cas9 recleavage of accurate repair products or both. While NU7441 suppressed production of $G F P^{+}$cells induced by a high amount of Cas9-gEJ $\mathrm{W}_{\mathrm{W}} 6$ at $0.25 \mu \mathrm{g}$ each, the inhibitor started to stimulate production of $G F P^{+}$cells when the amount of Cas 9 and $g E J_{W} 6$ was both reduced to $0.001 \mu \mathrm{g}$ (Fig $2 \mathrm{~b}, \mathrm{c}$ ). In contrast, at the $\mathrm{gEJ}_{\mathrm{W}} 7$ target, NU7441 did not alter the frequency of $G F P^{+}$cells induced by Cas9 and gEJW 7 at an amount ranging from $0.25 \mu \mathrm{g}$ to $0.0001 \mu \mathrm{g}$ (Fig $2 \mathrm{~d}, \mathbf{e}$ ). This further confirms that c-NHEJ is not involved in repair of Cas9-induced DSB at the $g E J_{W} 7$ target after the interference of target recleavage is minimized.

We then reassessed the c-NHEJ engagement at the 6 Cas9-sgRNA target sites when Cas9 recleavage of the regenerated target is prevented by lowering the transfection amount of Cas9-sgRNA. At the two sites targeted by $\mathrm{gEJ}_{\mathrm{W}} 4$ and $\mathrm{gEJ} \mathrm{J}_{\mathrm{W}} 6$ with the transfection amount of Cas9-sgRNA at $0.001 \mu \mathrm{g}$, Cas9-induced indels were also reduced as expected (Fig 2f). DNA-PKcs inhibition and XRCC4 deletion did not suppress production of Cas9-induced $G F P^{+}$cells any more or even reversed to stimulation at the $\mathrm{gEJ}_{\mathrm{W}} 4$ and $\mathrm{gEJ} \mathrm{J}_{\mathrm{W}} 6$ targets but remained to exert no effect on the level of Cas9-induced $G F P^{+}$cells at the $\mathrm{gEJ} \mathrm{J}_{\mathrm{W}} 3$ or $\mathrm{gEJ} \mathrm{J}_{\mathrm{W}} 7$ site (Fig 2f). In fact, $X R C C 4$ deletion elevated the frequency of Cas9-induced $G F P^{+}$cells by $59.6 \pm 14.2 \%$ $(P<0.05)$ at the $\mathrm{gEJ}_{\mathrm{W}} 4$ target and $81.5 \pm 24.5 \%(P<0.05)$ at the $\mathrm{gEJ}_{\mathrm{W}} 6$ target with $0.001 \mu \mathrm{g}$ of Cas9-sgRNA (Fig 2f), a reverse from reduction of Cas9-induced $G F P^{+}$cells by $58.1 \pm 3 \%$ and $60.4 \pm 2.4 \%$ respectively at these two targets with $0.25 \mu \mathrm{g}$ of Cas9-sgRNA (Fig 1e). These results again indicate that limiting Cas 9 recleavage could elicit the stimulatory effect of c-NHEJ inactivation on Cas9-induced indels.

Differently, at the $\mathrm{gEJ}_{\mathrm{C}} 5$ or $\mathrm{gEJ} \mathrm{J}_{\mathrm{W}} 5$ target, with the transfection amount of Cas9-sgRNA at $0.001 \mu \mathrm{g}$, DNAPKcs inhibition and XRCC4 deletion still inhibited the generation of Cas9-induced GFP ${ }^{+}$cells; but this inhibition was reduced (Fig $2 \mathrm{f}$ ). At the $\mathrm{gEJ}_{\mathrm{C}} 5$ target, $X R C C 4$ deletion reduced Cas9-induced $G F P^{+}$cells by 
$37.0 \pm 3.2 \%(P<0.001)$ with $0.001 \mu \mathrm{g}$ of Cas9-sgRNA, a smaller reduction than $55.8 \pm 2.6 \%(P<0.001)$ with $0.25 \mu \mathrm{g}$ of Cas9-sgRNA (Fig $2 \mathrm{f}$ vs. $1 \mathrm{e}$ ). At the $\mathrm{gEJ} \mathrm{W}_{\mathrm{W}} 5$ target, this reduction of $G F P^{+}$cells by XRCC4 deletion is $57.6 \pm 7.5 \%(P<0.001)$ with $0.001 \mu \mathrm{g}$ of Cas9-sgRNA but $69.2 \pm 1.5 \%(P<0.01)$ with $0.25 \mu \mathrm{g}$ of Cas9sgRNA (Fig $2 \mathrm{f}$ vs. 1e). This suggests that Cas9 recleavage could still abrogate the stimulatory effect of cNHEJ inactivation on Cas9-induced indels at the $\mathrm{gEJ}_{\mathrm{C}} 5$ or $\mathrm{gEJ}_{\mathrm{W}} 5$ target sites where limiting Cas 9 recleavage does not fully abolish the suppression of Cas9-induced indels by c-NHEJ inactivation. Similar to the $g E J_{W} 7$ target, no effect by c-NHEJ inactivation was detected at the gE $J_{W} 3$ target with neither $0.001 \mu \mathrm{g}$ nor $0.25 \mu \mathrm{g}$ of Cas9-sgRNA (Fig $2 \mathrm{f}$ and Fig 1e), suggesting no engagement of c-NHEJ at these two sites. Taken together, these results not only indicate that target recleavage by Cas 9 amplifies the mutagenicity of c-NHEJ in CRISPR/Cas9 genome editing, but also confirm that the involvement of cNHEJ varies significantly at different targets in repair of Cas9-induced DSBs after target recleavage by Cas9 is partially or fully prevented.

\section{Weakening target interaction of Cas9-sgRNA biases repair of Cas9-induced DSBs towards C-NHEJ}

To further determine whether c-NHEJ repair of Cas9-induced DSBs is influenced by target interaction of Cas9-sgRNA, we compare the c-NHEJ engagement at the same target by changing the interaction between Cas9-sgRNA and target DNA. In this setting, the effects of DNA sequences or chromatin structures are fixed and only target interaction is allowed to change. We mutated either sgRNA or SpCas 9 for two sites targeted by $\mathrm{gEJ}_{\mathrm{C}} 5$ and $\mathrm{gEJ} \mathrm{J}_{\mathrm{W}} 7$ in the NHEJ reporter to reduce Cas9-sgRNA target interaction. In consistent with previous observation that reducing Cas9-sgRNA target interaction generally lowered the efficiency of genome editing [30-33], induction of Cas9-induced $G F P^{+}$cells was less efficient with mismatched or truncated sgRNA variants (i.e. the C2A mismatch, the T15A mismatch and the truncated 16nt for $g E J_{C} 5$, and A1T, A4C and T15A for $g E J_{W} 7$ ) and with SpCas9 variants eSpCas9 and SpCas9-HF1, both of which were engineered to have less target interaction (i.e. lower binding affinity) and higher specificity to target DNA (Fig 3a). The sequences of the sgRNA variants are listed in Additional file 1, Fig S3a. As in Fig 1e, DNA-PKcs inhibition and XRCC4 deletion reduced Cas9-induced $G F P^{+}$cells respectively by $30.1 \%$ and $62.4 \%$ at the site targeted with $S p C a s 9-g E J_{C} 5$, again suggesting significant DNA recleavage by Cas9 (Fig 3a). In contrast, at the same target, the $\mathrm{gEJ}_{\mathrm{C}} 5$ variants $\mathrm{C} 2 \mathrm{~A}$ and $\mathrm{T} 15 \mathrm{~A}$ alleviated or even reversed this NU7441-mediated reduction, and the $\mathrm{gEJ}_{\mathrm{C}} 5$ variant $16 \mathrm{nt}$ and SpCas9-HF1 strongly reversed the reduction by XRCC4 deletion as the fold changes of NHEJ stimulation induced by DNA-PKcs inhibition or $X R C C 4$ deletion between these Cas9-sgRNA variants and the SpCas9-gEJ 5 20nt control were more than 1 and up to 5.1 (Fig 3a). At the site targeted by $\mathrm{gEJ}_{\mathrm{W}} 7$, neither DNA-PKcs inhibition nor $X R C C 4$ deletion had effect on the frequency of Cas9-induced $G F P^{+}$cells as shown in Fig 1a (Fig 3a), indicating no engagement of c-NHEJ at this site. However, the $\mathrm{gEJ}_{\mathrm{W}} 7$ mismatch variant T15A and SpCas9-HF1 allowed significant NU7441-mediated stimulation of Cas9-induced GFP ${ }^{+}$cells (Fig 3a). T15A, eSpCas9 and SpCas9-HF1 also elicited stimulatory effect of XRCC4 deletion on Cas9-induced $G F P^{+}$cells at the $\mathrm{gEJ}_{\mathrm{W}} 7$ target site as the fold changes of this NHEJ stimulation between the Cas9sgRNA variants and the SpCas9-gEJ 7 20nt control were up to 3.5-fold (Fig 3a). This suggests that in 
repair of Cas9-induced DSBs, the weaker the Cas9-sgRNA target interaction is, the more preferentially cNHEJ is engaged.

Using endogenous genomic loci, we also found that the editing efficiency with the mismatch variants of Cola $1 \mathrm{gC} 4$ (i.e. C1T and $\mathrm{G} 16 \mathrm{C}$ ) and Rosa26 gR4 (i.e. A1C and A16T) was reduced due to weaker target interaction (Fig 3b, c and Additional file 1, Fig S3b). Consistently, DNA-PKcs inhibition by NU7441 had minimal effect on Cas9-induced indels at the sites targeted by Cola1 gC4 and Rosa26 gR4 (Fig 1f, g), but stimulated Cas9-induced indels with the gC4 variant G16C and the gR4 variants A1C and A16T (Fig 3b, C and Additional file 1, Fig S3b). This again indicates that reducing Cas9-sgRNA target interaction promotes c-NHEJ. Taken together, these results suggest that weakened target interaction of Cas9-sgRNA increase bias toward c-NHEJ in repair of Cas9-induced DSBs.

\section{Weakening target interaction of Cas9-sgRNA enhances stimulatory effect of c-NHEJ inactivation on Cas9- induced HDR}

Consistently with previous studies [20-22], inactivation of c-NHEJ stimulates HDR induced by CRISPR nucleases as well as I-Scel (Fig 1 b, $\mathbf{c}$ and Additional file 1, Fig S1c). We expected that this stimulatory effect would be further enhanced if HDR were induced by Cas9-sgRNA variants with reduced target interaction, because reducing Cas9-sgRNA target interaction promotes c-NHEJ. We thus compared HDR induced by mutated Cas9-sgRNA between cells proficient and deficient in c-NHEJ. Due to reduced efficiency of DNA cutting, Cas9-induced HDR was generally less efficient with mismatched or truncated sgRNA variants (i.e. G1C, G2C and $17 \mathrm{nt}$ for $\mathrm{gHR}_{\mathrm{C}} 4$, and $\mathrm{A} 1 \mathrm{~T}, \mathrm{C} 2 \mathrm{~A}$ and $17 \mathrm{nt}$ for $\mathrm{gHR}_{\mathrm{C}} 2$ ) and SpCas9 variants eSpCas9, SpCas9-HF1 and xCas9 (i.e. xCas9-3.7), except eSpCas9-gHR $\mathrm{C}_{\mathrm{C}} 4$, SpCas9-gHR 2 17nt and xCas9-gHR 2 20nt (Fig 3d, e and Additional file 1, Fig S3c).

At the site targeted by $\mathrm{gHR}_{\mathrm{C}} 4$, as in Fig 1c, Cas9-induced HDR was not affected by DNA-PKcs inhibition, DNA-PKCs deletion or Ku80 deletion, but modestly stimulated by deletion of XRCC4, Cas9-induced HDR with the sgRNA variants G2C and 17nt was elevated by NU7441 (Fig 3d). Similarly, deletion of DNA-PKCS or Ku80 elicited stimulatory effect on Cas9-induced HDR with $\mathrm{gHR}_{\mathrm{C}} 4 \mathrm{G1C}$ and $17 \mathrm{nt}$, as well as with SpCas9-HF1 (Fig 3d). In addition, XRCC4 deletion stimulated Cas9-induced HDR with the $\mathrm{gHR}_{\mathrm{C}} 4$ variants (i.e. G1C, G2C and 17nt) and the SpCas9 variants SpCas9-HF1 and XCas9 by up to 4.3-fold (Fig 3d). At the site targeted by $\mathrm{gHR}_{\mathrm{C}} 2$, where Cas9-induced HDR was increased by DNA-PKcs inhibition or deletion of DNA-PKCS, Ku80 or XRCC4 as in Fig 1c, stimulation of Cas9-induced HDR by NU7441 was further enhanced with the SpCas9 variants such as eSpCas9 and SpCas9-HF1 (Fig 3e). This HDR stimulation for the SpCas 9 variants increased by 1.2- to 2 .2-fold as compared to the SpCas 9 control (Fig 3e). Deletion of DNA-PKCS, Ku80 or XRCC4 caused more stimulation of Cas9-induced HDR for SpCas9-gHR 2 C2A, eSpCas9-20nt and SpCas9-HF1-20nt as this HDR stimulation were enhanced by up to 4.9-fold (Fig 3e).

However, neither DNA-PKcs inhibition nor genetic inactivation of c-NHEJ by deletion of DNA-PKCs, Ku80 or XRCC4 stimulated more HDR induced by eSpCas9-gHR 4 20nt, SpCas9-gHR 2 A1T, SpCas9-gHR 2 17nt or xCas9-gHR 2 20nt than that induced by their respective SpCas9-20nt controls (Fig 3d, e). It appeared 
that HDR induced by these Cas9-sgRNA variants is as efficient as that by their SpCas9-20nt controls at their target sites (Fig 3d, e). It is possible that little is changed in the strength of target interaction or the efficiency of target cleavage between the SpCas9-20nt control and Cas9-sgRNA variants at these sites despite modification of SpCas9 or sgRNA. Taken together, these results above confirm that reducing target interaction of Cas9-sgRNA promotes c-NHEJ, providing the basis for the enhanced stimulatory effect of c-NHEJ inactivation on Cas9-induced HDR.

\section{Inactivation of c-NHEJ increases off-target activity of CRISPR/Cas9}

As mismatches in base pairing between sgRNA and off-target sites weaken the interaction of Cas9sgRNA with off-target sites, it is anticipated that c-NHEJ would be engaged proportionally more at offtarget sites than at on-target sites. In addition, target recleavage occurs less at off-target sites. Thus, inactivation of c-NHEJ would increase the engagement of a-NHEJ at off-target sites. As a-NHEJ is more error prone even for directly ligatable ends, inactivation of c-NHEJ leads to proportionally more mutNHEJ events and exacerbates off-target effects in CRISPR/Cas9 genome editing. To test this hypothesis, we analyzed the effects of DNA-PKcs inhibition and XRCC4 deletion on off-target activities of Cas 9 at 7 potential off-target sites for gPnpla3 and 6 potential off-target sites for gMertk and calculated the fold change of off-target effect due to DNA-PKcs inhibition and XRCC4 deletion. We found that both NU7441 and XRCC4 deletion slightly reduced on-target editing by Cas9-gPnpla3 and Cas9-gMertk by about 15$21 \%$, suggesting significant on-target DNA recleavage. In contrast, the frequencies of Cas9-induced indels at off-target sites were not reduced by either DNA-PKcs inhibition or XRCC4 deletion, but increased at many of these sites (Fig 4a, b, left). The fold change of off-target effect was more than 1 and even over 2 at some sites by c-NHEJ inactivation (Fig 4a, b, right). This suggests that inactivation of c-NHEJ aggravate off-target effect in CRISPR/Cas9 genome editing.

Chemical inhibition and genetic inactivation of c-NHEJ are often used to increase the efficiency of Cas9induced HDR-mediated gene knock-in or replacement [34-39]. Given that DNA-PKcs inhibition by NU7441 stimulated Cas9-induced HDR in the HDR reporter at the targets by $\mathrm{gHR}_{\mathrm{C}} 1$ and $\mathrm{gHR}_{\mathrm{C}} 2$ (Fig1b), we also performed off-target analysis for 6 potential off-target sites for Cas9-gHR 1 and Cas9-gHR 2 respectively. After NU7441 treatment, the frequencies of on-target indels induced by Cas9-gHR 1 and Cas9-gHR 2 were slightly lowered by $20-40 \%$, again indicating significant on-target DNA recleavage. Unlike on-target editing, the frequencies of Cas9-induced indels at the 6 off-target sites were not reduced by NU7441. Instead, these frequencies were stimulated by DNA-PKcs inactivation or XRCC4 deletion (Fig $4 \mathrm{c}$, d, left), and the fold change of off-target effect was elevated up to 2.5 (Fig $4 \mathrm{c}$, d, right). This again suggests that both chemical inhibition and genetic inactivation of c-NHEJ exacerbate off-target effects in CRISPR/Cas9 genome editing.

\section{Local transcription does not affect involvement of c-NHEJ in repair of Cas9-induced DSBs}

As weakening target interaction of Cas9-sgRNA promotes c-NHEJ engagement in repair of Cas9-induced DSBs, we wondered how the strength of Cas9-sgRNA target interaction controls the extent of c-NHEJ 
engagement. Since the Cas9-sgRNA complex remains bound to its target after DNA cleavage due to persistent target interaction of Cas9-sgRNA, it is possible that DNA ends are buried in the complex and do not fully elicit the DNA damage response (DDR) or engage any repair pathways before DNA end exposure [10-15]. While some ends are exposed by spontaneous dissociation of Cas9-sgRNA from cleaved target DNA and readily engage c-NHEJ, the others may require local transcription machinery to dislodge the target-bound Cas9-sgRNA complex [18]. The collision with local transcription machinery generates different DNA end configurations that may be unsuitable for binding c-NHEJ factors. If this was the case, we reasoned that the gene silencing activity (i.e. the transcription-blocking capability) of catalytically dead Cas9 (dCas9)-sgRNA at a given target would be negatively correlated with the extent of c-NHEJ participation in repair of Cas9-induced DSBs at the same site. Thus, using the single-copy GFP gene expression cassette integrated at the ROSA26 locus in the genome of mESC, we induced the GFP gene silencing at various sites by catalytically dead SpCas9 (dSpCas9) and also generated GFP ${ }^{-}$cells by SpCas9-induced GFP knock-out (KO) editing at these sites (Additional file 1, Fig S4a). We examined any potential correlation between dSpCas9-mediated gene silencing and c-NHEJ involvement in SpCas9induced GFPKO at the same sites. While dSpCas9-sgRNA exhibited variable gene silencing activities at many of these sites (Fig 5a), the effect of DNA-PKcs inhibition on GFP KO varied from no effect for $\mathrm{gG}_{\mathrm{C}} 1$, $\mathrm{gG}_{\mathrm{C}} 4, \mathrm{gG}_{\mathrm{C}} 7, \mathrm{gG}_{\mathrm{C}} 10, \mathrm{gG}_{\mathrm{C}} 14, \mathrm{gG}_{\mathrm{C}} 15$ and $\mathrm{gG}_{\mathrm{W}} 5$ to about 4 -fold stimulation for $\mathrm{gG}_{\mathrm{C}} 9$ and $\mathrm{gG}_{\mathrm{W}} 2$ among targets (Fig 5b and Additional file 1, Fig S4b). No apparent bias towards either template strand of transcription or non-template strand was detected in both transcription silencing by dSpCas9-sgRNA and DNA-PKcs involvement reflected by stimulation of SpCas9-induced GFPKO by NU7441 (Fig 5c). Importantly, no correlation was observed between transcription silencing by dSpCas9-sgRNA and stimulation of SpCas9-induced GFP KO by DNA-PKcs inhibition (Fig 5D; $P=0.78$ ), excluding the possibility that a collision with local transcription control the involvement of c-NHEJ in repair of Cas9-induced DSBs.

To further determine the effect of the collision between local transcription and Cas9-sgRNA on the engagement of c-NHEJ for Cas9-induced indels, we used catalytically dead Staphylococcus aureus Cas9 (dSaCas9)-sgRNA to block the translocating RNA polymerase (RNAP), preventing its collision with downstream site-specific DSBs induced by SpCas9 (Fig 5e). Among 6 sgRNAs tested for transcriptional blockage, only $\mathrm{gSaG}_{\mathrm{W}} 1$ and $\mathrm{gSaG}_{\mathrm{W}} 2$, in complex with dSaCas9, efficiently reduced gene expression by $26.7 \pm 4.5 \%(P<0.05)$ and $47.4 \pm 7.3 \%(P<0.01)$ respectively, indicating a strong capability of blocking RNAP (Fig 5e). The frequency of $G F P^{-}$cells induced by SpCas9-gG 4 and SpCas9-gG $\mathrm{W}_{\mathrm{W}} 5$ at a transfection amount of $0.125 \mu \mathrm{g}$ or $0.0005 \mu \mathrm{g}$ for each plasmid was not altered by DNA-PKcs inhibition with NU7441, and this non-effect was little changed by co-transfection with either $\mathrm{dSaCas9-gSaG}{ }_{W} 1$ or dSaCas9$\mathrm{gSaG}_{\mathrm{W}} 2$ (Fig 5f). This suggests that transcription blockage by dSaCas9-sgRNA (e.g. dSaCas9-gSaG 1 and dSaCas9-gSaG ${ }_{W}$ 2) would not affect the extent of c-NHEJ engagement in repair of SpCas9-induced DSBs and further confirms that a collision with local transcription do not control c-NHEJ engagement in repair of Cas9-induced DSBs.

\section{Local replication abolishes c-NHEJ engagement at Cas9-induced DSBs}


Like transcription, local DNA replication could also collide with Cas9-sgRNA that remains bound to the cleaved target and dislodge Cas9-sgRNA from the cleaved DNA, generating end configurations that may not be suitable for engaging c-NHEJ. Additionally, the collision with the replication fork occurs in S phase, where HDR is favored for replication-coupled DSB repair. Thus, to investigate whether collision with local DNA replication underlies the biased disengagement of C-NHEJ in repair of Cas9-induced DSBs at some target sites, we transfected HEK293 cells with a plasmid containing an SV40 origin-ATG-GFP-P2A-FLuc NHEJ reporter cassette, together with expression plasmids for SV40 large T antigen (LT), I-Scel or the SpCas9-gEJ 10 complex, and the Renilla luciferase (RLUC) gene as internal control. The expression of SV40 $L T$ drives bidirectional DNA replication via the SV40 origin, and the expression of I-Scel or SpCas9gEJW10 induces a site-specific DSB between the "Koz-ATG" and the "ATG-GFP-P2A-FLuc" (Fig 6a). Repair of I-Scel- or Cas9-induced DSBs mostly by c-NHEJ generate indels that can proportionally reframe the originally out-of-frame firefly luciferase (FLuc) gene in the NHEJ reporter plasmids to in-frame in the cells and induce synthesis of active firefly luciferase. The frequency of I-Scel- or Cas9-induced indels can thus be measured as a relative ratio of FLuc to RLuc by luminescence assays. Treatment with NU7441 reduced I-Scel-induced indels by $62.0 \pm 7.3 \%$ in this assay, but the level of this reduction was similar at $58.0 \pm 6.1 \%$ with the expression of SV40 $L T$ (Fig 6a), suggesting little effect of local DNA replication on IScel-induced indels. However, while Cas9-induced indels was also suppressed by $83.0 \pm 2.3 \%$ with NU7441, DNA replication initiated by SV40 LT significantly attenuated this repressive effect to $33.0 \pm 5.8 \%$ (Fig 6a). This suggests that local DNA replication driven by SV40 LT might inhibit the involvement of cNHEJ in repair of Cas9-induced DSBs.

We also wondered whether a collision with local DNA replication would favor HDR over c-NHEJ in repair of Cas9-induced DSBs by blocking c-NHEJ engagement, thus removing the stimulatory effect of DNAPKcs inhibition on Cas9-induced HDR. Using U2OS cells containing an integrated single-copy HDR reporter (Fig 6b), in which an SV40 origin is located between TrGFP and I-Scel-GFP, we analyzed the effect of DNA-PKcs inhibition by NU7441 on HDR induced by I-Scel and SpCas9. In consistent with the results from mESC (Fig 1b), NU7441 stimulated HDR induced by SpCas9 in complex with gHR $1, \mathrm{gHR}_{\mathrm{C}} 2, \mathrm{gHR}_{\mathrm{C}} 3$, $\mathrm{gHR}_{\mathrm{C}} 4$ and $\mathrm{gHR}_{\mathrm{C}} 5$ to different degrees, as well as by I-Scel (Fig $6 \mathrm{~b}$ and Additional file 1, Fig S5a), indicating variable but detectable engagement of the competing c-NHEJ pathway in repair of these I-Scelor Cas9-induced DSBs. After expression of SV40 LT, HDR induced by I-Scel, Cas9-gHR 2 , Cas9-gHR 3 and Cas9-gHR 4 were repressed in a gradual and dose-dependent manner (Fig $6 \mathrm{~b}$ and Additional file 1, Fig S5b, c). NU7441 stimulated I-Scel- or Cas9-induced HDR, and the expression of SV40 $L T$ attenuated this stimulation of I-Scel-induced HDR from 4.9-fold to 2.5-fold or even abolished the NU7441-induced stimulation of Cas9-induced HDR at a transfection amount of $0.032 \mu \mathrm{g}(1 / 25$ of total DNA transfected) for Cas9-gHR 4 and $0.16 \mu \mathrm{g}(1 / 5$ of total DNA transfected) for Cas9-gHR 2 (Fig $\mathbf{6 b}$ and Additional file 1, Fig S5b, c). This suggests that local DNA replication driven by SV40 LT could collide with both I-Scel and SpCas9-sgRNA after DNA cleavage to dislodge I-Scel and Cas9-sgRNA from its cleaved target and restrict the engagement of c-NHEJ in repair of exposed DSBs. 
By restricting c-NHEJ due to a collision with replication fork, DSB repair pathway choice would be biased toward HDR. To test this possibility, we used the HDR reporter to measure the bias between HDR and NHEJ in repair of the same DSB induced by SpCas9-sgRNA that was tightly bound with its target and by SpCas9-sgRNA variants with weakened target interaction. In the HDR reporter, repair of the same Cas9induced DSBs around the I-Scel site of I-Scel-GFP by HDR generates the "WT GFP", whereas NHEJ generates "mutant $G F P$ " due to disruption of the I-Scel site (Fig 6c). We can separate these two repair outcomes in mESC by nested PCR and evaluate the HDR bias (i.e. the ratio of HDR to total edited) by deep sequence analysis. After HDR and NHEJ induced by SpCas9-gHR 4 in mESC, we found the HDR bias was nearly 3-fold lower with gHR 4 variants (G1C and 17nt) than with gHR $\mathrm{H}_{\mathrm{C}} 4$ (Fig 6d), indicating a reduced HDR preference when the interaction of SpCas9-sgRNA to its target is weakened. At the site targeted by $\mathrm{gHR}_{\mathrm{C}} 2$, where the HDR stimulation by DNA-PKcs inhibition was fully abolished in U2OS cells by the expression of SV40 $L T$ at a transfection amount of $0.16 \mu \mathrm{g}$ (Fig 6b), SV40 $L T$ expression at the same transfection amount increased the HDR bias by nearly 2 -fold (Fig 6d), indicating a shift of the repair pathway from NHEJ to HDR. Therefore, for Cas9-sgRNA target sites where c-NHEJ is disfavored in repair of Cas9-induced DSBs, it is likely that Cas9-sgRNA at these sites may have a higher probability for collision with local DNA replication after DNA cleavage due to persistent target interaction. Cas9-induced replication-coupled DSBs are subsequently generated with particular end configurations in S phase and favor HDR over c-NHEJ for their repair.

\section{Palindromic fusion of sister chromatids arises from collision of Cas9-sgRNA at cleaved targets with DNA replication}

While spontaneous dissociation of Cas9-sgRNA from cleaved DNA results in a conventional two-ended DSB, DNA replication that releases Cas9-sgRNA from its cleaved target may generate a three-ended DSB, with the leading strand likely forming a blunt end on one sister chromatid and the lagging strand a 3'overhanging end with long ssDNA on the other sister chromatid (Fig 6e). These two ends each can rejoin with the other blunt end of the DSB, or have a potential to directly ligate with each other, the latter generating a palindromic chromosome from sister chromatid fusion (SCF) and potentially promoting chromatid breakage-fusion-bridge (BFB) cycles [40-43] (Fig 6e). Because neither DNA-PKcs nor Ku80 is engaged at Cas9-induced DSBs at the gHR 4 target site for repair in the HDR reporter in mESC (Fig 1 b, c), it is likely that Cas9-gHR 4 at this site may collide with a replication fork after DNA cleavage, generating a three-ended DSB and allowing subsequent fusion of two sister chromatids and production of a palindromic chromosome. Because the product contains palindromic DNA sequence surrounding the junctions, a single primer could in theory be annealed to both the leading strand template and the newly synthesized lagging strand in the repair product for PCR amplification. However, no PCR products were detected from repair of Cas9-induced DSBs at the $\mathrm{gHR}_{\mathrm{C}} 4$ target site in the HDR reporter in $\mathrm{mESC}$ and U2OS cells with a single primer e.g. TF1, TF2 or TF3 (data not shown), likely due to the interference in PCR amplification by palindromic DNA sequences [44]. Given potentially asymmetric sequence deletion at the junction of SCF, we thus paired a distal primer to the break (TF2 or TF3) with the most proximal primer TF1 to minimize the length of palindromic DNA sequence in PCR amplification of repair products 
induced by SpCas9-gHR 4 in the HDR reporter and detected PCR bands over 250bp in mESC (Fig $6 f$ and Additional file 1, Fig S6a). In U2OS cells, these PCR bands were detected only after expression of SV40 $L T$, suggesting replication-coupled generation of three-ended DSBs and fusion of newly duplicated sister chromatids at the SpCas9-gHR 4 cleavage site (Fig 6 f and Additional file 1, Fig S6a). This is consistent with the observation that DNA-PKcs inhibition stimulates HDR induced by Cas9-gHR 4 in U2OS cells, but neither in mESC nor in U2OS cells highly expressing SV40 $L T$.

To further confirm that the PCR bands for these repair products were indeed fusions of sister chromatids via end ligation of Cas9-induced DSBs, we first cloned PCR products into a plasmid for Sanger sequencing. Among 40 clones for PCR bands with TF1 and TF2, 17 were from mESC and the rest from U2OS cells. Among 31 clones for PCR bands with TF1 and TF3, 29 were from mESC and the rest from U2OS cells. Sanger sequencing revealed only two sequence variations in each PCR band: DL251R6 and DL268R1 for the PCR band with TF1 and TF2 and DL231R5 and DL386R45 for the PCR band with TF1 and TF3 (Fig $6 \mathrm{~g}$ and Additional file 1, Fig S6b). They all contained some GFP sequences inverted around the break site but no palindromic GFP sequences, indicating that SCF may occur but palindromic sequences may be lost during repair or may not be amplified by PCR (Additional file 2, Table S1). In addition, the deletion length in each sequence was distinctly asymmetric surrounding the break point, long at $231 \mathrm{bp}, 251 \mathrm{bp}, 268 \mathrm{bp}$ or $386 \mathrm{bp}$ at one direction and short at $1 \mathrm{bp}, 5 \mathrm{bp}, 6 \mathrm{bp}$ or $45 \mathrm{bp}$ at the other direction (Fig $\mathbf{6 g}$ and Additional file 1, Fig S6b). It is likely that the collision between DNA replication and Cas9-sgRNA could generate long ssDNA at the lagging strand end and little or no ssDNA overhang at the leading strand end. Long ssDNA could be easily degraded, generating long deletion. PCR targeted amplicon sequencing also confirmed inverted GFP sequences with no palindromic fragments around Cas9-induced DSBs, but with more junction sequence variations (Additional file 1, Fig S7a, b). Taken together, these results suggest that three-ended DSBs could be generated from release of Cas9-sgRNA at some cleaved targets upon encountering local DNA replication, resulting in inverted duplication via endjoining of sister chromatids.

\section{Discussions}

In in vitro biochemical assays, the target-binding affinity of Cas9-sgRNA is primarily determined by the interactions of Cas9-sgRNA with its target $[2,10,45]$. As Cas9-sgRNA binds DNA targets with varying affinities and remains bound for variable time even after DNA cleavage, one key issue often ignored in the development and application of CRISPR/Cas9 genome editing is possible effects of Cas9-sgRNA target interaction on DSB repair pathway choice in repair of Cas9-induced DSBs. These effects could hamper our efforts in predicting and improving the efficiency and specificity of CRISPR/Cas9 genome editing. In this study, we demonstrate that target interaction of Cas9-sgRNA modulates c-NHEJ involvement in repair of Cas9-induced DSBs, shaping the choices of repair pathway that differ among targets with varying strength of Cas9-sgRNA target interaction (Fig $\mathbf{6 h}$ ). It also helps explain why inactivation of c-NHEJ by chemical or genetic approaches enhance HDR-mediated CRISPR/Cas9 genome editing at some sites [34-39], not at others $[46,47]$. Even at a same target, due to different strength and persistence of Cas9- 
sgRNA target interaction, Cas9-sgRNA could be dissociated from cleaved DNA either spontaneously or by local transcription or DNA replication (Fig $\mathbf{h}$ ), exposing Cas9-induced DSBs with different end configurations for specific repair pathways. Weaker target interaction may permit more frequent, spontaneous dissociation or transcription-mediated dissociation of Cas9-sgRNA from its cleaved targets. DSBs exposed in this way can readily engage c-NHEJ. In contrast, stronger and more persistent target interaction delays DSB exposure and increases the probability of a collision between Cas9-sgRNA and local replication forks, generating DSB ends that disfavor c-NHEJ and potentially inducing inverted ligation of sister chromatids (Fig $\mathbf{6 h}$ ). This may lead to extensive structural abnormalities in chromosomes. Therefore, this regulation of DSB repair pathway choice not only provides insight into how on-target gross chromosomal rearrangements is generated in CRISPR/Cas9 genome editing, but also is potentially a new source for the heterogeneity of mutation profiles in CRISPR/Cas9 genome editing (Fig $6 h)$.

Like any other types of DSBs, a pathway choice for repair of Cas9-induced DSBs is influenced by many factors such as cell cycle stage, nucleotide composition and configuration of DNA ends, surrounding chromatin structure and local DNA metabolism [7]. Owing to the innate complexity of DSB repair pathways and the interplay of the factors that regulate DSB repair pathway choice, repair products in CRISPR/Cas9 genome editing are highly heterogeneous in mammalian cells, making it difficult to accurately predict mutation profiles or readily isolate favorable genomic edits in genome editing. Structural and biochemical studies have demonstrated that DSB induction by Cas9-sgRNA is distinct as compared to ionized radiation (IR), radiomimetic drugs and other DNA endonucleases $[2,10-14,48,49]$. Prior to DNA cleavage, Cas9-sgRNA binds to its target via the base pairing of sgRNA with target DNA strand and the interactions of Cas9 with both sgRNA and target DNA, and initiates the R-loop formation. The R-loop formation in turn activates cleavage of target DNA strand and non-target DNA strand by Cas9. After DSB induction, Cas9-sgRNA remains bound to the cleaved DNA products, concealing the DSBs from access by the DDR and repair machineries [10-15]. In this case, exposure of DSBs is a prerequisite for DSB recognition and repair. Previous studies indicate that Cas9-sgRNA could be released from cleaved targets either spontaneously or by forces such as DNA replication, transcription or chromatin remodeling in eukaryotic cells, exposing DSBs [8,9,16-19]. Because different forms of Cas9-sgRNA dissociation after DNA cleavage may modify Cas9-induced DSBs with different end configurations, it is possible that target interaction of Cas9-sgRNA may modulate DSB repair pathway choices by influencing the residence duration of Cas9-sgRNA at cleaved DNA and dissociation of Cas9-sgRNA from it. This regulation may add a new layer of control over DSB repair pathway choices and additional complexity into generation and prediction of mutations in CRISPR/Cas9 genome editing.

It has been previously shown that dSpCas9-sgRNA could block translocating RNAP at some targets, thus repressing gene expression [50]. Further, sgRNAs targeting the coding DNA strand of transcription generally demonstrate better gene silencing than sgRNAs targeting the template strand $[18,50]$. This raises a possibility that Cas9-sgRNA bound to its cleaved target could encounter translocating RNAP and be removed from cleaved DNA by a collision with transcription in a strand-biased manner. While the collision with transcription on template strand might facilitate genome editing more efficiently than on 
the coding strand [18,50], our data indicate that c-NHEJ involvement is not altered by the collision of Cas9-sgRNA with transcription no matter which strand the sgRNA is paired with. It is likely that throughout the cell cycle, transcriptional collision, like spontaneous dissociation, may expose DSBs with clean ends that can be recognized and rejoined easily by c-NHEJ factors. Mechanical perturbations such as DNA torsion and DNA stretching imposed by chromatin remodeling may also destabilize the Cas9sgRNA-DNA complex and dislodge Cas9-sgRNA from the cleaved DNA [51-53]. Assuming the cell cycle would not be altered by these mechanical perturbations, the end configurations of Cas9-induced DSBs exposed in these cases would remain unchanged and not alter DSB repair pathway choice.

However, replication-coupled dissociation of Cas9-sgRNA from cleaved DNA is restricted to the S phase of the cell cycle and generates three-ended DSBs, which appear to reject c-NHEJ for repair. In these threeended DSBs, the staggered end with a long 3'-ssDNA overhang may not engage c-NHEJ factors such as Ku70/Ku80, and the availability of sister chromatids can further promote HDR, antagonizing c-NHEJ. Therefore, when using Cas9-induced DSBs at individual sites to study regulation of DSB repair pathway choices, we should avoid generalization unless it is taken into consideration how Cas9-sgRNA interacts with its target and is released from it after DNA cleavage. In addition, repair of the three-ended DSBs provides an opportunity for the DNA ends of two sister chromatids to rejoin, not only creating a palindromic chromosome with two centromeres or no centromere [42,43], but also leaving the third end for potential translocation. Both dicentric and acentric palindromic chromosomes are unstable and serves as a potential source for chromothripsis and complex chromosomal rearrangements including large deletions and insertions at the target site [40-43,54]. Therefore, this study identifies a potential mechanism underlying on-target chromosomal rearrangements previously detected in CRISPR/Cas 9 genome editing [55-59]. In contrast to forced dissociation by DNA replication in S phase, spontaneous dissociation might occur in different stages of the cell cycle, where the DSB repair pathway choice differs partly due to availability of repair factors or substrates for different repair pathways. However, in a population of asynchronous cells, the effect of the cell cycle stage offsets one another among different cells and does not appear to be significant. Although it remains poorly understood how Cas9-sgRNA is spontaneously released from cleaved targets, the strength of target interaction of Cas9-sgRNA may affect spontaneous dissociation of Cas9-sgRNA from cleaved DNA and residence duration of Cas9-sgRNA. Taken together, target interaction of Cas9-sgRNA should be integrated with a network of regulators into a decision point for final DSB repair pathway choices at different targets or even at a same target, generating different sets of repair products and contributing to the heterogeneity of mutation profiles in CRISPR genome editing.

Off-target effects are a serious problem in CRISPR/Cas9 genome editing and have greatly limited clinical use of this technology [30]. Due to single or multiple mismatches between sgRNA and off-target DNA, the interaction of Cas9-sgRNA with off-target sites is weaker than that at the on-target site. As a result, the DNA binding affinity of Cas9-sgRNA at an off-target site is much weaker in general, and the residence duration could be shorter [30]. Thus, Cas9-sgRNA at off-target sites, despite being less efficient in DNA cleavage, is dissociated from the cleaved DNA more frequently in a spontaneous manner, exposing DSBs that are more likely to engage c-NHEJ. In addition, because DNA recleavage occurs less at off-target sites 
than at on-target sites, c-NHEJ, which is innately accurate in repair of Cas9-induced DSBs, generates even less mutagenic repair events at off-target sites. Therefore, while inactivation of c-NHEJ by chemical or genetic approaches is often used to enhance HDR-mediated CRISPR/Cas9 genome editing [34-39], our study revealed that this strategy generate more off-target mutations and cause stronger off-target effects. However, this stimulation of off-target effect was often ignored in CRISPR/Cas9 genome editing [34-38], and should be addressed when we use the strategy to enhance HDR-mediated CRISPR/Cas9 genome editing.

Because the tight target binding of Cas9-sgRNA is excessive for genome editing at some sites, reducing this target binding to some degree may not affect on-target activity but help significantly mollify offtarget effects [31]. Various strategies have been designed to remove the excessive target binding and improve the specificity of the modified Cas9-sgRNA [30]. These strategies include truncating 20-nt spacer of a sgRNA to 17-18 nt and mutating the Cas9 residues that are important for non-specific interactions of Cas9 with non-target strand of DNA and the RNA-DNA hybrid [31-33]. We found that when these truncated sgRNA or Cas9 variants such as SpCas9-HF1 and eSpCas9, as compared with wild-type SpCas9, are used in CRISPR/Cas9 genome editing, inactivation of c-NHEJ by chemical inhibitors or genetic modifications may enhance genome editing including NHEJ-mediated gene KO or HDR-mediated knock-in. However, these c-NHEJ inactivation approaches still exert their effects globally in genome editing mediated by these variants, thus increasing off-target activities. Therefore, in either case of Cas9sgRNA or its high-fidelity variants, a better strategy is needed to locally inhibit c-NHEJ while causing no additional off-target effects in CRISPR/Cas9 genome editing.

\section{Conclusions}

Herein, we demonstrated that target interaction of Cas9-sgRNA is a new regulator of DSB repair pathway choice in CRISPR/Cas9 genome editing. Indeed, involvement of c-NHEJ varies in repair of Cas9-induced DSBs at different target sites. Weakening target interaction of Cas9-sgRNA biases the repair pathway choice towards c-NHEJ. Thus, inactivation of c-NHEJ elicits more stimulatory effect on Cas9-induced HDR at a target where target interaction of Cas9-sgRNA is weakened. In addition, due to weaker binding at off-target sites, the off-target activity of Cas9-sgRNA is exacerbated by c-NHEJ inactivation, which is often used to promote HDR-based CRISPR genome editing. Our study also revealed a mechanism by which target interaction of Cas9-sgRNA controls DSB repair pathway choices at Cas9-induced DSBs. In particular, at sites with stronger target interaction or by extension, longer target-residence duration of Cas9-sgRNA, a collision of Cas9-sgRNA with local DNA replication would dislodge Cas9-sgRNA from cleaved DNA, generating three-ended DSBs unsuitable for c-NHEJ repair. During repair of these threeended DSBs, palindromic ligation of sister chromatids could occur at the break site, potentially leading to on-target gross chromosomal rearrangements, an editing outcome that has been widely reported as a serious concern in applications of CRISPR/Cas9 genome editing [55-59]. Therefore, target interaction of Cas9-sgRNA could be an important contributor to significant on-target and off-target mutation variations in CRISPR/Cas9 genome editing by modulating repair pathway choices in repair of Cas9-induced DSBs. 


\section{Materials And Methods}

\section{Plasmids}

The expression plasmids for truncated and mismatched sgRNAs were constructed as described [31], and the expression plasmids for SpCas9, SpCas9 variants eSpCas9, SpCas9-HF1 and xCas9-3.7, and d SpCas 9 were constructed previously $[32,33,60]$. The sgRNA target sequences and respective mutations for SpCas9 and SaCas9 are listed in Additional file 2, Table S2. The HDR reporter plasmid was previously constructed $[23,61]$. To generate the reporter plasmid GFP-P2A-FLuc for replication fork-SpCas 9 collision assays, the P2A-Firefly luciferase (FLuC) gene was fused to C-terminal of GFP in the sGEJ reporter previously established [26]. Due to an SV40 replication origin originally present in the SGEJ reporter, DNA replication can be induced by expression of SV40 LT in the GFP-P2A-FLuc collision reporter.

\section{Cell lines}

HEK293 cells were cultured in Dulbecco's modified Eagle medium (DMEM) containing $10 \%$ fetal bovine serum, $1 \%$ penicillin-streptomycin and $2 \mathrm{mM}$ L-glutamine. The NHEJ reporter mESC, the HDR reporter mESC and the HDR reporter U2OS cells were previously established and cultured as described before $[23-26,62]$. To generate the $G F P^{+}$cell lines for GFP KO experiments, mESC harboring the NHEJ reporter were transfected with expression plasmids for SpCas9-g-I-Scel and $G F P^{+}$cells were cloned, expanded and determined by fluorescence-activated cell sorting (FACS) using the Beckman Coulter CytoFLEX flow cytometer. Isogenic $X R C C 4^{+/+}$and $X R C C 4^{-/-}$mESC containing the HDR reporter were established previously [24]. DNA-PKCs ${ }^{-/-}$and $K u 80^{-/-} \mathrm{HDR}$ reporter mESC along with isogenic wild-type clones were generated by the paired Cas9-sgRNA method as previously described [5].

\section{Transfection and DSB repair reporter assays}

Transfection of mESC was done with Lipofectamine 2000 (Invitrogen) in 24-well plates as previously described [25,61]. For U2OS or HEK293 cells transfection, $1.0 \times 10^{5}$ cells were seeded on a 24-well plate and grown to $80-95 \%$ confluence. $0.8 \mu \mathrm{g}$ total DNA were transfected by Lipofectamine 2000 . Cells harboring the NHEJ or HDR reporter were transfected with pcDNA3b-I-Scel or the expression plasmids for SpCas9-sgRNA or SaCas9-sgRNA as previously described $[25,61]$.

In dSaCas9-sgRNA transcription blockage experiments, GFP ${ }^{+} \mathrm{mESC}$ were transfected with the expression plasmids for SpCas9-sgRNA, together with the expression plasmids for dSaCas9-sgRNA. In replication fork-dSpCas9 collision experiments, cells were transfected with the expression plasmids for I-Scel or SpCas9-sgRNA and the SV40 LT, together with the GFP-P2A-FLuc reporter plasmid as needed. If necessary, cells were treated with DNA-PKcs inhibitor NU7441 (TopScience Cat\# T6276) at $6 \mathrm{~h}$ posttransfection. NU7441 was replaced with a fresh addition of the drug the next day. GFP ${ }^{+}$and $G F P^{-}$cells were determined by FACS at $72 \mathrm{~h}$ and $96 \mathrm{~h}$ respectively post-transfection. The frequencies of NHEJ, HDR and genome editing were calculated after being corrected with background readings and normalized with transfection efficiencies as described before [25]. 
To evaluate the effect of Cas9 dosage on NHEJ, NHEJ reporter cells were transfected with a varying amount of Cas9-sgRNA each at $0.25 \mu \mathrm{g}, 0.1 \mu \mathrm{g}, 0.01 \mu \mathrm{g}, 0.001 \mu \mathrm{g}$ and $0.0001 \mu \mathrm{g}$. Cells transfected were treated with $2.5 \mu \mathrm{M}$ NU7441 and analyzed by FACS 3 days post-transfection.

\section{GFP fluorescence measurement for CRISPRi in mESC}

$G F P^{+}$reporter cells were transiently transfected with $0.25 \mu \mathrm{g}$ each of dCas9 and sgRNA expression plasmids in 24-well plates. Cells were analyzed at $96 \mathrm{~h}$ post transfection for GFP fluorescence intensity using Beckman Coulter CytExpert 2.0 normalized with mCherry transfection efficiency (TE). The GFP fluorescence intensity of cells transfected with each dCas9-sgRNA was calculated as below:

I (sgRNA): GFP intensity of cells expressing dCas9-sgRNA; I (sgRNAmeasured): GFP intensity of cells after transfecting with dCas9-sgRNA; I (CTRL measured): GFP intensity of cells after transfecting with dCas9-control sgRNA.

\section{Luciferase assay}

HEK293 cells were transiently transfected with GFP-P2A-Luciferase-based NHEJ reporter plasmids together with the expression plasmids for I-Scel or Cas9-sgRNA. The reporter was supplied at $0.025 \mu \mathrm{g}$ in each well of 24-well plates. At $48 \mathrm{~h}$ post transfection, cells were harvested and analyzed with the Dual Luciferase Reporter Assay system (Promega). All assays were done in triplicates and all values normalized for transfection efficiency against Renilla luciferase activities as internal control.

\section{PCR targeted amplicon sequencing}

For analysis of targeted genome editing at endogenous genome loci, cells were collected after NHEJ induced by Cas9-sgRNAs. Genomic DNA (gDNA) was isolated from these cells using a gDNA purification kit (Axygen). The targeted regions were PCR-amplified with respective primers listed in Additional file 2, Table S3. The Illumina deep sequencing was performed at Novogene Co. Ltd and subsequent data analysis was performed as previously described [25].

\section{Off-target analysis}

Potential off-target sites were identified using the latest version of the CRISPR Off-Target prediction website (http:// crispor.tefor.net/). All potential sites were ranked by an off-target hit score, and highranked potential sites were selected. Off-target sites were amplified by PCR with primers listed in Additional file 2, Table S3 after gDNA extraction from cells transfected with Cas9-sgRNA at 3 d posttransfection. Off-target editing efficiency was determined by Illumina deep sequencing. The off-target rate was determined as the ratio of off-target to on-target mutagenesis levels.

\section{Three-ended DSB repair analysis}


HDR reporter mESC were transfected with Cas9-sgRNA and harvested $2 \mathrm{~d}$ post-transfection. For HDR reporter U2OS cells, $0.008 \mu \mathrm{g}$ of the SV40 LT plasmid in $0.8 \mu \mathrm{g}$ of total DNA was simultaneously transfected to initiate replication. gDNA was collected and the palindromic DNA sequences were amplified by touchdown PCR with primers listed in Additional file 2, Table S3. PCR amplicons were subcloned into CE Entry vector (Vazyme C114-02) and analyzed by Sanger sequencing. Deep sequencing of PCR amplicons was also performed and their repair junctions were characterized by bioinformatics analysis.

\section{Statistical analysis}

Two-tailed Student's paired or unpaired t-test was used for statistical analysis of repair frequencies, i.e. the frequencies of Cas9- and I-Scel-induced GFP ${ }^{+}$cells, Cas9-induced GFP- cells or Cas9-induced indels. Two-tailed Student's unpaired t-test also allowed statistical analysis of comparison between two groups of sgRNAs targeting template strand of transcription or non-template strand, respectively. One-way ANOVA with post-hoc Dunnett's multiple comparison test was performed for fold change of NHEJ alteration and HDR stimulation by inactivation of c-NHEJ between Cas9-sgRNA variants and their respective SpCas9-sgRNA controls and for fold change of off-target effect between NU7441 and DMSO, and between $\mathrm{XRCC}^{+/+}$and $\mathrm{XRCC4^{-/- }}$ cells. Correlation between transcription silencing and the NHEJ increase was determined by linear regression analysis.

\section{Declarations}

\section{Declaration of interests}

The authors declare that they have no known competing financial interests or personal relationships that could have appeared to influence the work reported in this paper.

\section{Ethics approval and consent to participate}

Not applicable

\section{Consent for publication}

Not applicable

\section{Availability of data and materials}

Deep sequencing raw data are available in the Sequence Read Archive (SRA) under accession number PRJNA726333 (https://www.ncbi.nlm.nih.gov/sra/ PRJNA726333).

\section{Competing interests}

The authors declare that they have no competing interests. 


\section{Funding}

This work is funded by the National Natural Science Foundation of China (No. 31870806 and No. 31671385 to A.Y.X., and No. 32071439 to Y.L.F), the Natural Science Foundation of Zhejiang Province (LQ20C050004 to S.C.L) and Fundamental Research Funds for the Central Universities in China (2019QNA7031 to Y.L.F).

\section{Authors' contributions}

S-C.L., Y-L.F. and X-N.S. generated DNA constructs and cell lines, conducted repair reporter assays and genome editing experiments, and performed bioinformatics analysis. R-D.C. conducted replication collision experiments and data analysis. Q.L., J-J.X., J-F.X., G-Q.C., Y.Y., S-M.X., and H.L. assisted with generation of DNA constructs and cell lines. C.L., H-D.L. and A-Y.X. assisted with bioinformatics analysis. A-Y.X. conceived the project and supervised the study. S-C.L., Y-L.F., X-N.S., R-D.C. and A-Y.X. analyzed and discussed the data, and S-C.L., Y-L.F. and A-Y.X. wrote the manuscript.

\section{Acknowledgements}

We thank members of the Xie lab for helpful discussions and the Core Facilities at Hua Jia Chi Campus, Zhejiang University School of Medicine, for technical support. We thank J. Hu at Peking University for the gift of expression plasmids for Cas 9 variants and J.T. Wu for critically reading the manuscript.

\section{References}

1. Hsu PD, Lander ES, Zhang F. Development and applications of CRISPR-Cas9 for genome engineering. Cell. 2014;157(6):1262-78.

2. Jiang F, Doudna JA. CRISPR-Cas9 structures and mechanisms. Annu Rev Biophys. 2017;46:50529.

3. Jasin M, Haber JE. The democratization of gene editing: Insights from site-specific cleavage and double-strand break repair. DNA Repair (Amst). 2016;44:6-16.

4. Boboila C, Alt FW, Schwer B. Classical and alternative end-joining pathways for repair of lymphocytespecific and general DNA double-strand breaks. Adv Immunol. 2012;116:1-49.

5. Guo T, Feng Y-L, Xiao J-J, Liu Q, Sun X-N, Xiang J-F, et al. Harnessing accurate non-homologous end joining for efficient precise deletion in CRISPR/Cas9-mediated genome editing. Genome Biol. 2018;19(1):170.

6. Bétermier M, Bertrand P, Lopez BS. Is non-homologous end-joining really an inherently error-prone process? PLoS Genet. 2014;10(1):e1004086.

7. Symington LS, Gautier J. Double-strand break end resection and repair pathway choice. Annu Rev Genet. 2011;45:247-71.

8. Feng Y, Liu S, Chen R, Xie A. Target binding and residence: a new determinant of DNA double-strand break repair pathway choice in CRISPR/Cas9 genome editing. J Zhejiang Univ Sci B. 2021;22(1):73- 
86.

9. Xue C, Greene EC. DNA repair pathway choices in CRISPR-Cas9-mediated genome editing. Trends Genet. 2021;37(7):639-56.

10. Sternberg SH, Redding S, Jinek M, Greene EC, Doudna JA. DNA interrogation by the CRISPR RNAguided endonuclease Cas9. Nature. 2014;507(7490):62-7.

11. Richardson CD, Ray GJ, DeWitt MA, Curie GL, Corn JE. Enhancing homology-directed genome editing by catalytically active and inactive CRISPR-Cas9 using asymmetric donor DNA. Nat Biotechnol. 2016;34(3):339-44.

12. Knight SC, Xie L, Deng W, Guglielmi B, Witkowsky LB, Bosanac L, et al. Dynamics of CRISPR-Cas9 genome interrogation in living cells. Science. 2015;350(6262):823-6.

13. Ma H, Tu L-C, Naseri A, Huisman M, Zhang S, Grunwald D, et al. CRISPR-Cas9 nuclear dynamics and target recognition in living cells. J Cell Biol. 2016;214(5):529-37.

14. Kim S, Kim D, Cho SW, Kim J, Kim J-S. Highly effıcient RNA-guided genome editing in human cells via delivery of purified Cas9 ribonucleoproteins. Genome Res. 2014;24(6):1012-9.

15. Brinkman EK, Chen T, de Haas M, Holland HA, Akhtar W, van Steensel B. Kinetics and fidelity of the repair of Cas9-induced double-strand DNA breaks. Mol Cell. 2018;70(5):801-13.e6.

16. Verkuijl SA, Rots MG. The influence of eukaryotic chromatin state on CRISPR-Cas9 editing efficiencies. Curr Opin Biotechnol. 2019;55:68-73.

17. Wang AS, Chen LC, Wu RA, Hao Y, McSwiggen DT, Heckert AB, et al. The histone chaperone FACT induces Cas 9 multi-turnover behavior and modifies genome manipulation in human cells. Mol Cell. 2020;79(2):221-33.e5.

18. Clarke R, Heler R, MacDougall MS, Yeo NC, Chavez A, Regan M, et al. Enhanced bacterial immunity and mammalian genome editing via RNA-polymerase-mediated dislodging of Cas9 from doublestrand DNA breaks. Mol Cell. 2018;71(1):42-55.e8.

19. Vrtis KB, Dewar JM, Chistol G, Wu RA, Graham TGW, Walter JC. Single-strand DNA breaks cause replisome disassembly. Mol Cell. 2021;81(6):1309-18.e6.

20. Pierce AJ, Hu P, Han M, Ellis N, Jasin M. Ku DNA end-binding protein modulates homologous repair of double-strand breaks in mammalian cells. Genes Dev. 2001;15(24):3237-42.

21. Allen C, Kurimasa A, Brenneman MA, Chen DJ, Nickoloff JA. DNA-dependent protein kinase suppresses double-strand break-induced and spontaneous homologous recombination. Proc Natl Acad Sci U S A. 2002;99(6):3758-63.

22. Delacôte F, Han M, Stamato TD, Jasin M, Lopez BS. An xrcc4 defect or Wortmannin stimulates homologous recombination specifically induced by double-strand breaks in mammalian cells. Nucleic Acids Res. 2002;30(15):3454-63.

23. Rass E, Chandramouly G, Zha S, Alt FW, Xie A. Ataxia telangiectasia mutated (ATM) is dispensable for endonuclease I-Scel-induced homologous recombination in mouse embryonic stem cells. J Biol Chem. 2013;288(10):7086-95. 
24. Xie A, Hartlerode A, Stucki M, Odate S, Puget N, Kwok A, et al. Distinct roles of chromatin-associated proteins MDC1 and 53BP1 in mammalian double-strand break repair. Mol Cell. 2007;28(6):1045-57.

25. Feng YL, Xiang JF, Liu SC, Guo T, Yan GF, Feng Y, et al. H2AX facilitates classical non-homologous end joining at the expense of limited nucleotide loss at repair junctions. Nucleic Acids Res. 2017;45(18):10614-33.

26. Xie A, Kwok A, Scully R. Role of mammalian Mre11 in classical and alternative nonhomologous end joining. Nat Struct Mol Biol. 2009;16(8):814-8.

27. Bindra RS, Goglia AG, Jasin M, Powell SN. Development of an assay to measure mutagenic nonhomologous end-joining repair activity in mammalian cells. Nucleic Acids Res. 2013;41(11):e115.

28. Richardson CD, Ray GJ, Bray NL, Corn JE. Non-homologous DNA increases gene disruption efficiency by altering DNA repair outcomes. Nat Commun. 2016;7:12463.

29. Wolfs JM, Hamilton TA, Lant JT, Laforet M, Zhang J, Salemi LM, et al. Biasing genome-editing events toward precise length deletions with an RNA-guided TevCas9 dual nuclease. Proc Natl Acad Sci USA. 2016;113(52):14988-93.

30. Kim D, Luk K, Wolfe SA, Kim J-S. Evaluating and enhancing target specificity of gene-editing nucleases and deaminases. Annu Rev Biochem. 2019;88:191-220.

31. Fu Y, Sander JD, Reyon D, Cascio VM, Joung JK. Improving CRISPR-Cas nuclease specificity using truncated guide RNAs. Nat Biotechnol. 2014;32(3):279-84.

32. Kleinstiver BP, Pattanayak V, Prew MS, Tsai SQ, Nguyen NT, Zheng Z, et al. High-fidelity CRISPR-Cas9 nucleases with no detectable genome-wide off-target effects. Nature. 2016;529(7587):490-5.

33. Slaymaker IM, Gao L, Zetsche B, Scott DA, Yan WX, Zhang F. Rationally engineered Cas9 nucleases with improved specificity. Science. 2016;351(6268):84-8.

34. Chu VT, Weber T, Wefers B, Wurst W, Sander S, Rajewsky K, et al. Increasing the efficiency of homology-directed repair for CRISPR-Cas9-induced precise gene editing in mammalian cells. Nat Biotechnol. 2015;33:543-8.

35. Maruyama T, Dougan SK, Truttmann MC, Bilate AM, Ingram JR, Ploegh HL. Increasing the efficiency of precise genome editing with CRISPR-Cas9 by inhibition of nonhomologous end joining. Nat Biotechnol. 2015;33(5):543-8.

36. Lin S, Staahl BT, Alla RK, Doudna JA. Enhanced homology-directed human genome engineering by controlled timing of CRISPR/Cas9 delivery. Elife. 2014;3:e04766.

37. Yeh CD, Richardson CD, Corn JE. Advances in genome editing through control of DNA repair pathways. Nat Cell Biol. 2019;21(12):1468-78.

38. Canny MD, Moatti N, Wan LCK, Fradet-Turcotte A, Krasner D, Mateos-Gomez PA, et al. Inhibition of 53BP1 favors homology-dependent DNA repair and increases CRISPR-Cas9 genome-editing efficiency. Nat Biotechnol. 2018;36(1):95-102.

39. Gavande NS, VanderVere-Carozza PS, Pawelczak KS, Mendoza-Munoz P, Vernon TL, Hanakahi LA, et al. Discovery and development of novel DNA-PK inhibitors by targeting the unique Ku-DNA 
interaction. Nucleic Acids Res. 2020;48(20):11536-50.

40. Umbreit NT, Zhang CZ, Lynch LD, Blaine LJ, Cheng AM, Tourdot R, et al. Mechanisms generating cancer genome complexity from a single cell division error. Science. 2020;368(6488):eaba0712.

41. Kagaya K, Noma-Takayasu N, Yamamoto I, Tashiro S, Ishikawa F, Hayashi MT. Chromosome instability induced by a single defined sister chromatid fusion. Life Sci Alliance. 2020;3(12):e202000911.

42. Tanaka $\mathrm{H}$, Watanabe $\mathrm{T}$. Mechanisms underlying recurrent genomic amplification in human cancers. Trends Cancer. 2020;6(6):462-77.

43. Svetec Miklenić M, Svetec IK. Palindromes in DNA - A risk for genome stability and implications in cancer. Int J Mol Sci. 2021;22(6):2840.

44. Hommelsheim CM, Frantzeskakis L, Huang M, Ülker B. PCR amplification of repetitive DNA: a limitation to genome editing technologies and many other applications. Sci Rep. 2014;4:5052.

45. Jinek M, Chylinski K, Fonfara I, Hauer M, Doudna JA, Charpentier E. A programmable dual-RNAguided DNA endonuclease in adaptive bacterial immunity. Science. 2012;337(6096):816-21.

46. Zhang JP, Li XL, Li GH, Chen W, Arakaki C, Botimer GD, et al. Efficient precise knockin with a double cut HDR donor after CRISPR/Cas9-mediated double-stranded DNA cleavage. Genome Biol. 2017;18(1):35.

47. Yang D, Scavuzzo MA, Chmielowiec J, Sharp R, Bajic A, Borowiak M. Enrichment of G2/M cell cycle phase in human pluripotent stem cells enhances HDR-mediated gene repair with customizable endonucleases. Sci Rep. 2016;6:21264.

48. Gallagher DN, Haber JE. Repair of a Site-specific DNA cleavage: Old-school lessons for Cas9mediated gene editing. ACS Chem Biol. 2018;13(2):397-405.

49. Anders $C$, Niewoehner O, Duerst A, Jinek M. Structural basis of PAM-dependent target DNA recognition by the Cas 9 endonuclease. Nature. 2014;513(7519):569-73.

50. Qi LS, Larson MH, Gilbert LA, Doudna JA, Weissman JS, Arkin AP, et al. Repurposing CRISPR as an RNA-guided platform for sequence-specific control of gene expression. Cell. 2013;152(5):1173-83.

51. Ivanov IE, Wright AV, Cofsky JC, Aris KDP, Doudna JA, Bryant Z. Cas9 interrogates DNA in discrete steps modulated by mismatches and supercoiling. Proc Natl Acad Sci USA. 2020;117(11):5853-60.

52. Newton MD, Taylor BJ, Driessen RPC, Roos L, Cvetesic N, Allyjaun S, et al. DNA stretching induces Cas9 off-target activity. Nat Struct Mol Biol. 2019;26(3):185-92.

53. Szczelkun MD, Tikhomirova MS, Sinkunas T, Gasiunas G, Karvelis T, Pschera P, et al. Direct observation of R-loop formation by single RNA-guided Cas9 and Cascade effector complexes. Proc Natl Acad Sci USA. 2014;111(27):9798-803.

54. Ly P, Cleveland DW. Rebuilding chromosomes after catastrophe: Emerging mechanisms of chromothripsis. Trends Cell Biol. 2017;27(12):917-30.

55. Zuccaro MV, Xu J, Mitchell C, Marin D, Zimmerman R, Rana B, et al. Allele-specific chromosome removal after Cas9 cleavage in human embryos. Cell. 2020;183(6):1650-64.e15. 
56. Burgio G, Teboul L. Anticipating and identifying collateral damage in genome editing. Trends in Genetics. 2020;36(12):905-14.

57. Kosicki M, Tomberg K, Bradley A. Repair of double-strand breaks induced by CRISPR-Cas9 leads to large deletions and complex rearrangements. Nat Biotechnol. 2018;36(8):765-71.

58. Adikusuma F, Piltz S, Corbett MA, Turvey M, McColl SR, Helbig KJ, et al. Large deletions induced by Cas9 cleavage. Nature. 2018; 560(7717):E8-E9.

59. Leibowitz ML, Papathanasiou S, Doerfler PA, Blaine LJ, Sun L, Yao Y, et al. Chromothripsis as an ontarget consequence of CRISPR-Cas9 genome editing. Nat Genet. 2021; 53(6):895-905.

60. Hu JH, Miller SM, Geurts MH, Tang W, Chen L, Sun N, et al. Evolved Cas9 variants with broad PAM compatibility and high DNA specificity. Nature. 2018;556(7699):57-63.

61. Xie A, Puget N, Shim I, Odate S, Jarzyna I, Bassing CH, et al. Control of sister chromatid recombination by histone H2AX. Mol Cell. 2004;16(6):1017-25.

62. Chandramouly G, Kwok A, Huang B, Willis NA, Xie A, Scully R. BRCA1 and CtIP suppress long-tract gene conversion between sister chromatids. Nat Commun. 2013;4:2404.

\section{Figures}




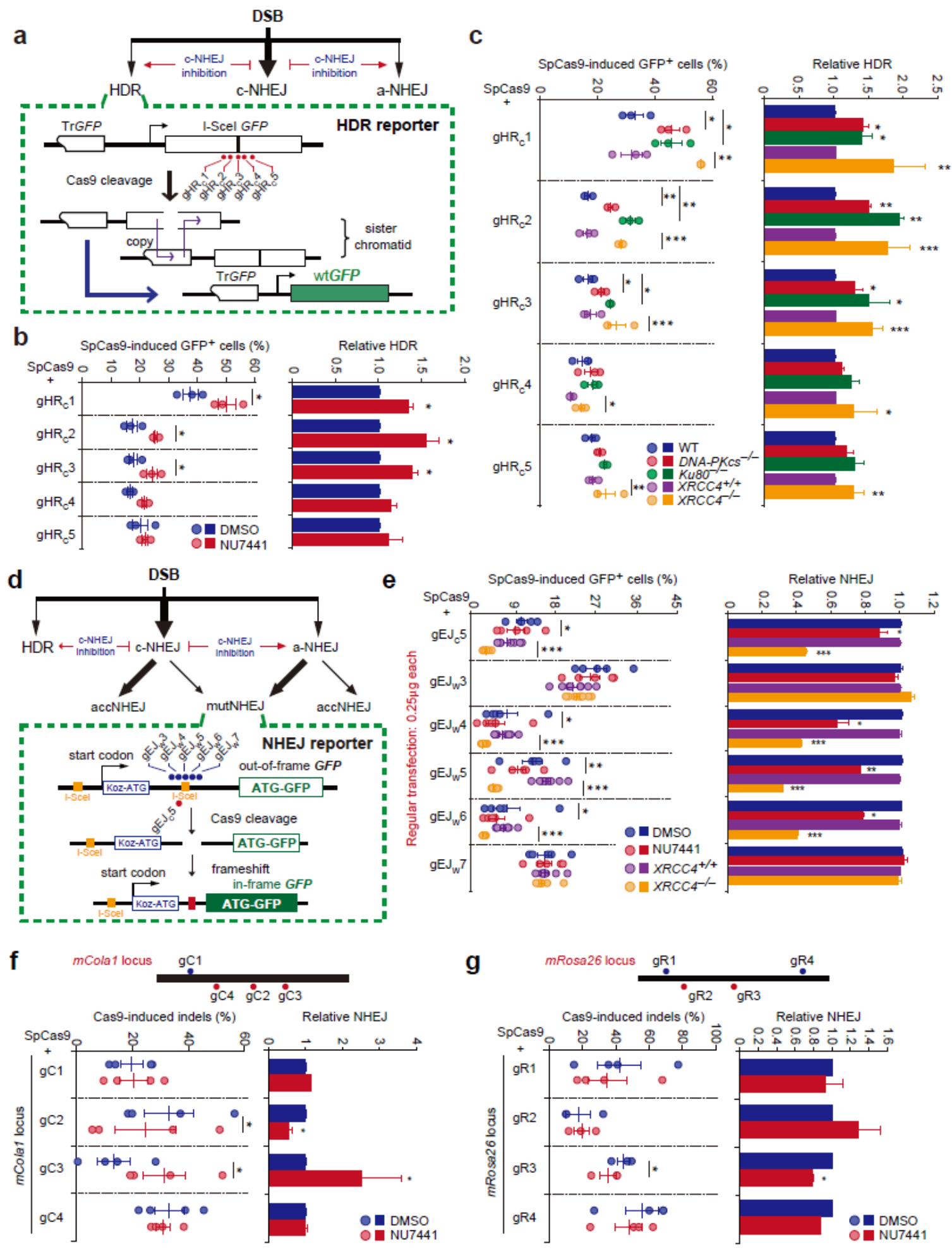

\section{Figure 1}

Involvement of c-NHEJ varies widely in repair of Cas9-induced DSBs. a Schematic of the HDR reporter with 5 sgRNAs. Repair of Cas9-induced DSBs by HDR between sister chromatids can generate $G F P^{+}$cells. Inhibition of c-NHEJ is expected to promote HDR. $\mathbf{b}$ Effects of DNA-PKcs inhibition on SpCas9-induced HDR in mESC transfected with individual Cas9-sgRNA. Left: Frequencies of SpCas9-induced $G F P^{+}$cells; Right: Relative HDR after normalizing DMSO treatment to 1.0. c Effects of DNA-PKCS, Ku80 or XRCC4 
deficiency on SpCas9-induced HDR in mESC transfected with individual Cas9-sgRNA. Left: Frequencies of SpCas9-induced $G F P^{+}$cells; Right: Relative HDR after normalizing both WT cells and $\mathrm{XRCC4}^{+/+}$cells to 1.0. d Schematic of the NHEJ reporter with 6 sgRNAs and their target sites indicated. Repair of Cas9induced DSBs by c-NHEJ or a-NHEJ generates accurate NHEJ (accNHEJ) products indistinguishable from undamaged targets and mutagenic NHEJ (mutNHEJ) products represented by $\mathrm{GFP}^{+}$cells. Inhibition of cNHEJ promotes a-NHEJ. e Effect of DNA-PKcs inhibition and XRCC4 deletion on SpCas9-induced NHEJ in mESC transfected with individual Cas9-sgRNA. Left: Frequencies of SpCas9-induced GFP ${ }^{+}$cells; Right: Relative NHEJ after normalizing both DMSO treatment and $X R C C 4^{+/+}$cells to $1.0 . \mathbf{f}, \mathbf{g}$ Cells were transfected with SpCas9-sgRNA expression plasmids and treated with DMSO or NU7441. Four different sites of the Cola 1 (f) and Rosa26 (g) locus were targeted by 4 sgRNAs indicated. The efficiency of SpCas9-induced genome editing (left) was calculated as ratios of edited reads to total reads from targeted Illumina sequencing and normalized by transfection efficiency. Relative SpCas9-induced NHEJ (right) was calculated by normalizing the editing efficiency with DMSO treatment to 1.0. Each circle indicates one independent experiment, each in triplicates. Columns indicate the mean \pm S.E.M of at least three independent experiments. Significance was detected by two-tailed Student's t-test and indicated by * for $P<0.05$, ** for $P<0.01$ and *** for $P<0.001$.
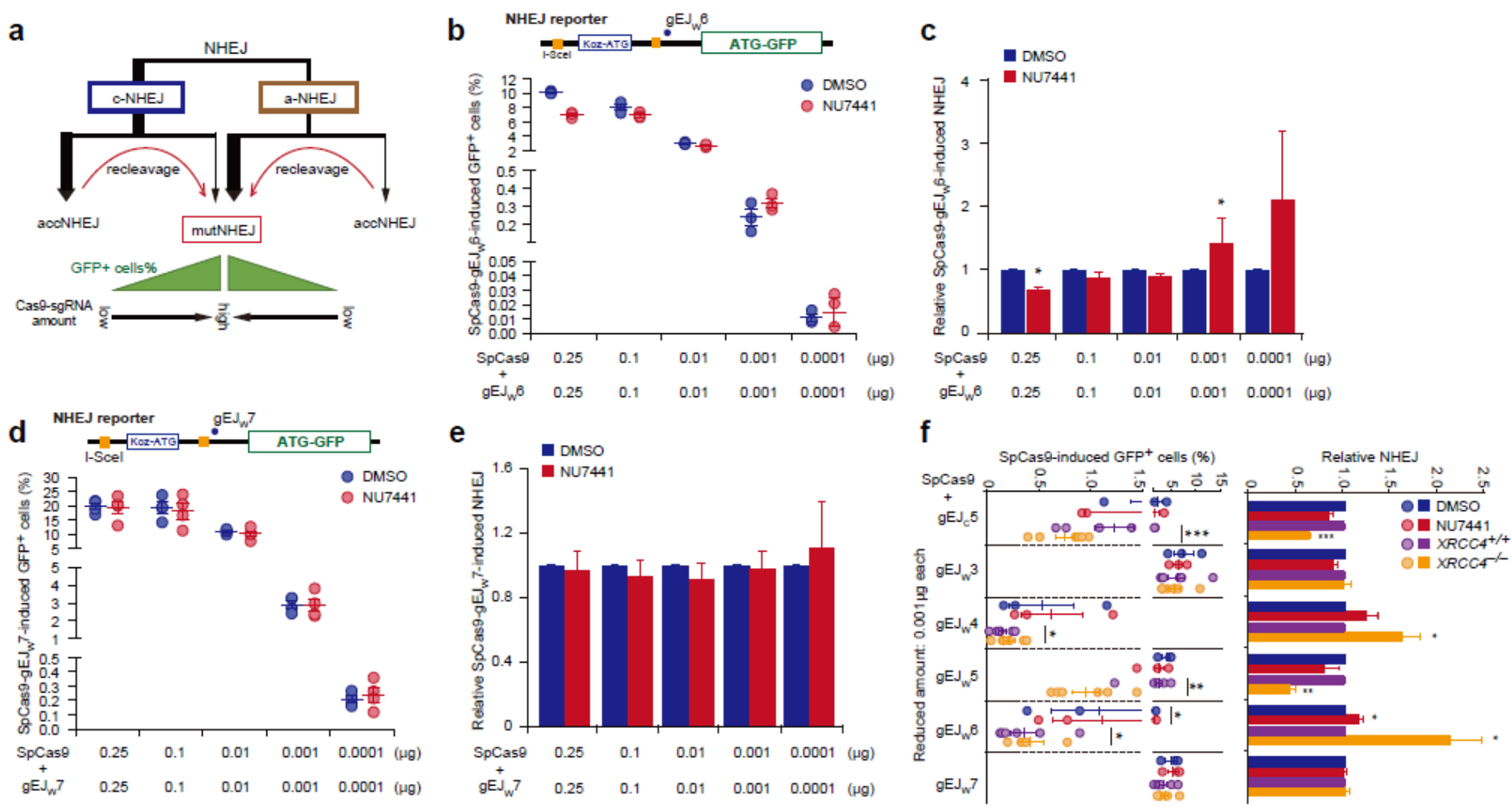

Figure 2

Cas9 recleavage increases c-NHEJ-mediated mutations. a Model for enrichment of mutNHEJ products promoted by frequent SpCas9 recleavage with increased amount of Cas9-sgRNA transfected. With sufficient amount of SpCas9-sgRNA, accNHEJ products could be recleaved until mutNHEJ products are 
generated, resulting in enrichment of mutNHEJ products. bee Effect of DNA-PKcs inhibition on NHEJ induced by varying amount of SpCas9-sgRNA. NHEJ reporter mESC were transfected with varying amount of expression plasmids for SpCas9-gEJ ${ }_{W} 6(\mathbf{b}, \mathbf{c})$ or SpCas9-gEJ 7 (d, e) as indicated and treated with DMSO or NU7441. Frequencies of SpCas9-induced GFP ${ }^{+}$cells $(\mathbf{b}, \mathbf{d})$ were measured by FACS at $3 \mathrm{~d}$ post-transfection and relative SpCas9-induced NHEJ was calculated by normalizing DMSO treatment to 1.0 (c, e). $\mathbf{f}$ Frequencies of $G F P^{+}$cells (left) and relative NHEJ (right) induced by SpCas9-sgRNA at $0.001 \mu \mathrm{g}$ each, $1 / 250$ of the regular amount $(0.25 \mu \mathrm{g}$ each) transfected into mESC. Each circle indicates one independent experiment, each in triplicates, and the mean of at least three independent experiments is also indicated. Columns indicate the mean \pm S.E.M. Statistical significance was detected by two-tailed Student's t-test: $*, P<0.05 ; * \star, P<0.01$ and $* * * \quad P<0.001$. 

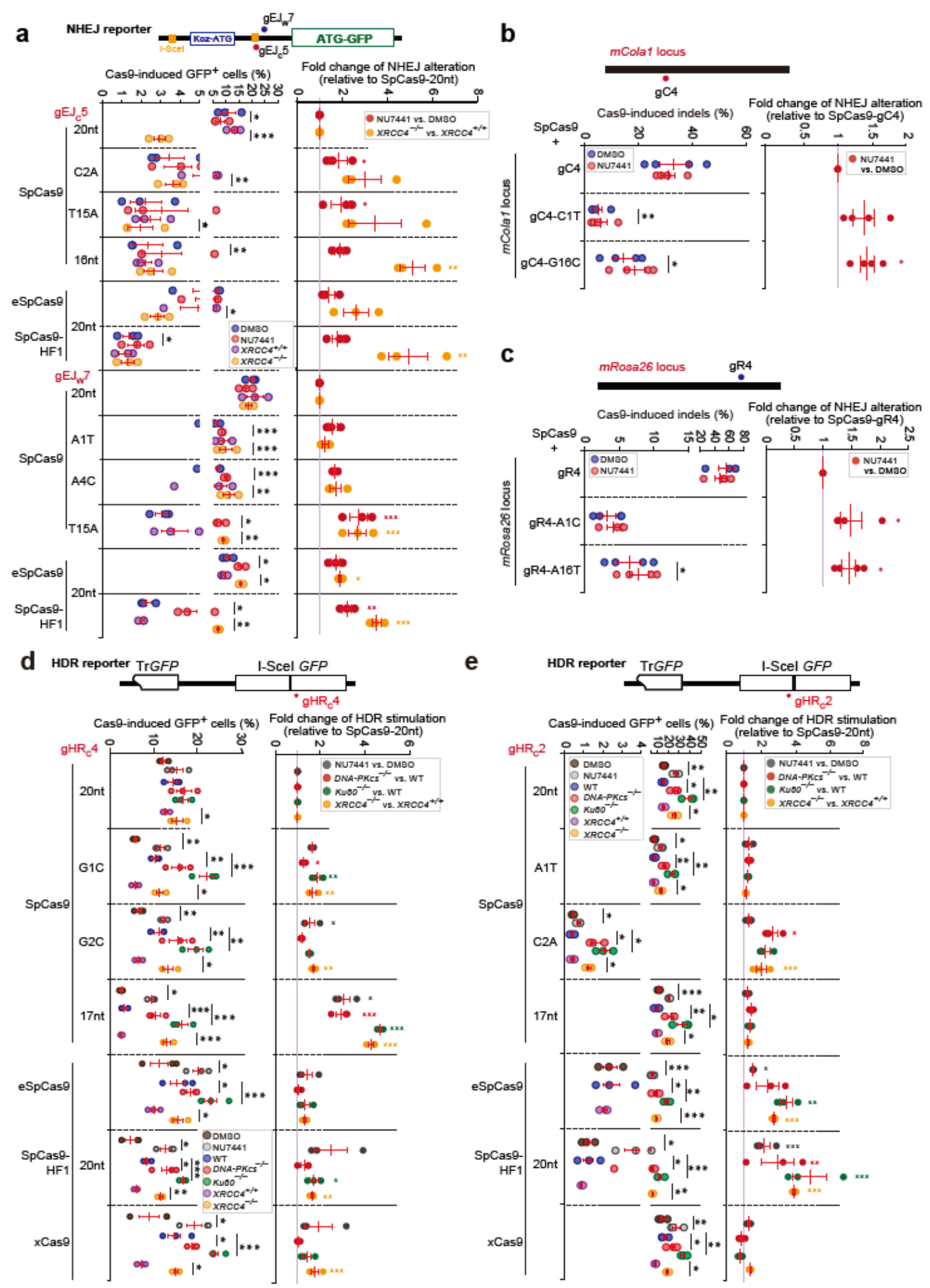

\section{Figure 3}

Reduced target-binding affinity of Cas9-sgRNA shifts the pathway bias towards c-NHEJ in repair of Cas9induced DSBs. a Effects of DNA-PKcs inhibition and XRCC4 deletion on SpCas9-induced NHEJ in mESC transfected with individual SpCas9-sgRNA and its variants as indicated. Left: Frequencies of SpCas9induced $G F P^{+}$cells; Right: Fold change of NHEJ alteration induced by NU7441 or XRCC4 deletion relative to the SpCas9-20nt control, i.e. the ratio of NHEJ change induced by NU7441 or XRCC4 deletion for each 
SpCas9-sgRNA variant to that for the SpCas9-20nt control. $\mathbf{b}, \mathbf{c}$ Effects of DNA-PKcs inhibition on NHEJmediated genome editing at endogenous loci Cola1 (b) and Rosa26 (c) in mESC transfected with individual SpCas9-sgRNA and its variants as indicated. The frequencies of Cas9-induced indels (left) were calculated as ratios of edited reads to total reads from targeted Illumina sequencing and normalized by transfection efficiency. The fold change of NHEJ alteration induced by NU7441 relative to the SpCas920nt control (right) was calculated as the ratio of NHEJ change induced by NU7441 for each SpCas9sgRNA variant to that for the SpCas9-sgRNA control. d, e Effects of DNA-PKcs inhibition and DNA-PKCS, $K u 80$ or XRCC4 deficiency on Cas9-induced HDR in mESC transfected with Cas9-gHR 4 (d), Cas9-gHR 2

(e) and its variants as indicated. Left: Frequencies of Cas9-induced $G F P^{+}$cells; Right: Fold change of HDR stimulation induced by NU7441 or deletion of DNA-PKCS, Ku80 or XRCC4 relative to the SpCas9-20nt control. This fold change was calculated as the ratio of HDR stimulation induced by NU7441 or deletion of DNA-PKCS, Ku80 or XRCC4 for each SpCas9-sgRNA variant to that for the SpCas9-20nt control. Each circle indicates one independent experiment, each in triplicates, and the mean of at least three independent experiments is also indicated. Columns indicate the mean \pm S.E.M. Statistical significance was detected by two-tailed Student's paired t-test for frequencies of Cas9-induced GFP ${ }^{+}$cells or Cas9induced indels and by one-way ANOVA followed by post-hoc Dunnett's test for fold changes of NHEJ alteration or HDR stimulation: ${ }^{*}, P<0.05 ; * \star,, P<0.01$ and $* \star * ~ P<0.001$. 

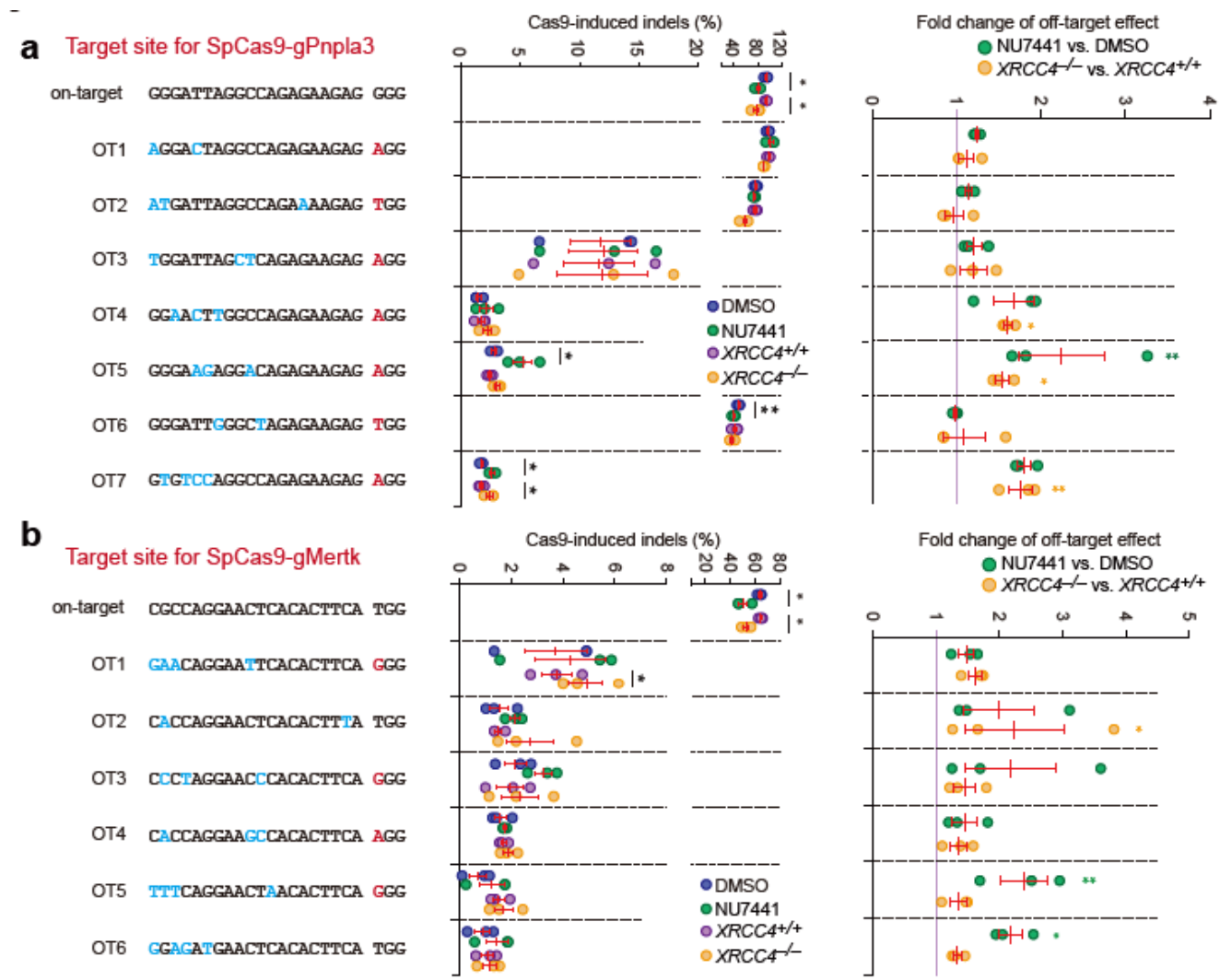

C

Target site for SpCas9-gHR 1

on-target TCGAGCTGAAGGGCATCGTA GGG

OT1 GGGAGCTGAAGGGCCTCGTA GGG

OT2 ACTAGCTGAAGaGCATTGTA GGG

ОT3 TGGGGCTGAAGGGCATCGCA TGG

OT4 gagagctgaAgGcCATCATA tGG

OT5 TCTAGCTGITGGGCATGGTA GGG

OT6 TCTGGCTCAAGGGCAACGTA AGG

d

Target site for SpCas9-gHR 2

on-target AGGGCATCGIAGGGATAACA GGG

OT1 AGgGCACTGAaGgGatAaCA TGG

OT2 ATGGCATCCTAGagataACA CGG

ОT3 TGGGiAgAgtaggGataACA AGG

OT4 TGagCATCGTAGgGatTaCA GGG

OT5 AGGGCATGGCAGGGATAACA AAG

OT6 AaGGCCTGCTAGgGatAACA GGG
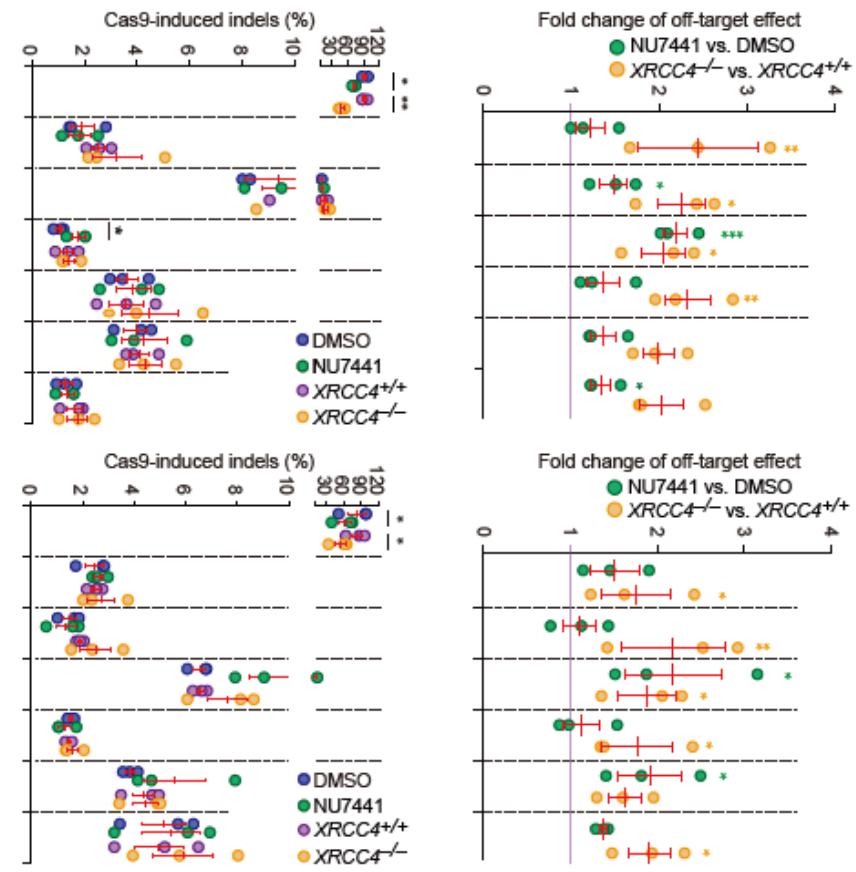

\section{Figure 4}

DNA-PKcs inhibition and XRCC4 deletion aggravate off-target effect in CRISPR/Cas9 genome editing. $X R C C 4^{+/+} \mathrm{HDR}$ reporter $\mathrm{mESC}$ were used for transfection with SpCas9 in complex with gPnpla3 targeting Pnpla3 (a), gMertk targeting Mertk (b), gHR 1 (c) and gHR 2 (d) both targeting the HDR reporter. At $6 \mathrm{~h}$ post transfection, cells were treated with DMSO or NU7441. At $72 \mathrm{~h}$ post-transfection, gDNA was isolated and the indel frequency at on-target and selected off-target sites was measured by amplicon deep 
sequencing and calculated as the ratio of edited reads to total reads normalized by transfection efficiency. In an independent set of experiments, isogenic $X R C C 4^{+/+}$and $X R C C 4^{-/-} \mathrm{HDR}$ reporter mESC were transfected and the indel frequency at on-target and selected off-target sites was similarly measured. Fold change of off-target effect after treatment of NU7441 or deletion of XRCC4 was calculated as the ratio of the indel frequency with treatment of NU7441 or in $X R C C 4^{-/-}$cells to that with DMSO or in $\mathrm{XRCC}^{+/+}$cells at each off-target site, respectively. Each circle indicates one independent experiment, and the mean of these independent experiments is also indicated. Error bars indicate S.E.M. Statistical analysis was performed by two-tailed Student's paired t-test for frequencies of Cas9-induced indels and by one-way ANOVA followed by post-hoc Dunnett's test for fold changes of off-target effect between NU7441 and DMSO, and between $\mathrm{XRCC4}^{+/+}$and $\mathrm{XRCC4}^{-/-}$. * for $\mathrm{P}<0.05$ and $* *$ for $\mathrm{P}<0.01$. 
a

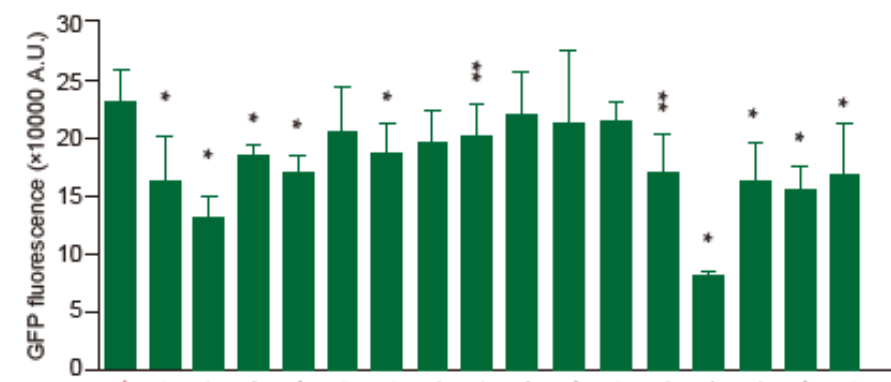

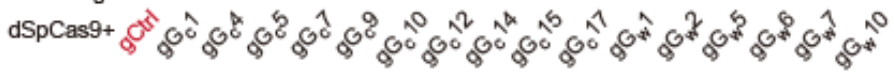

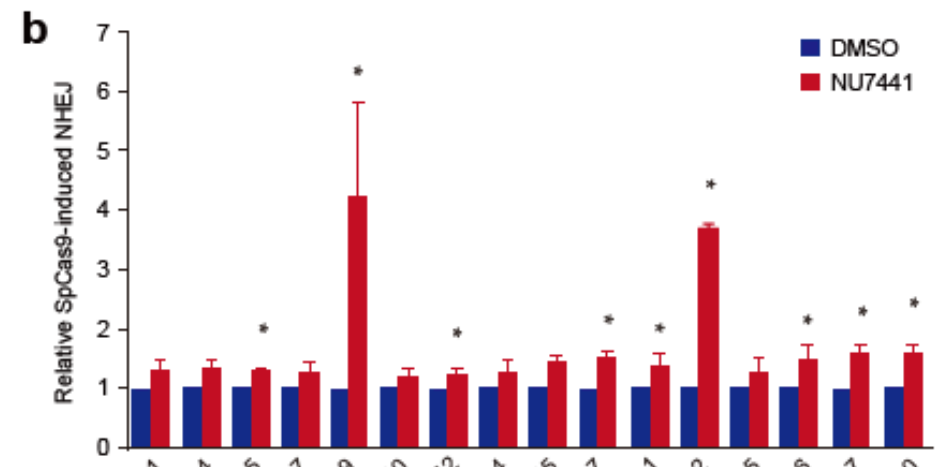

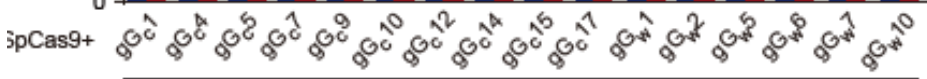

SpCas9-sgRNA transfected $(0.001+0.001 \mu \mathrm{g})$
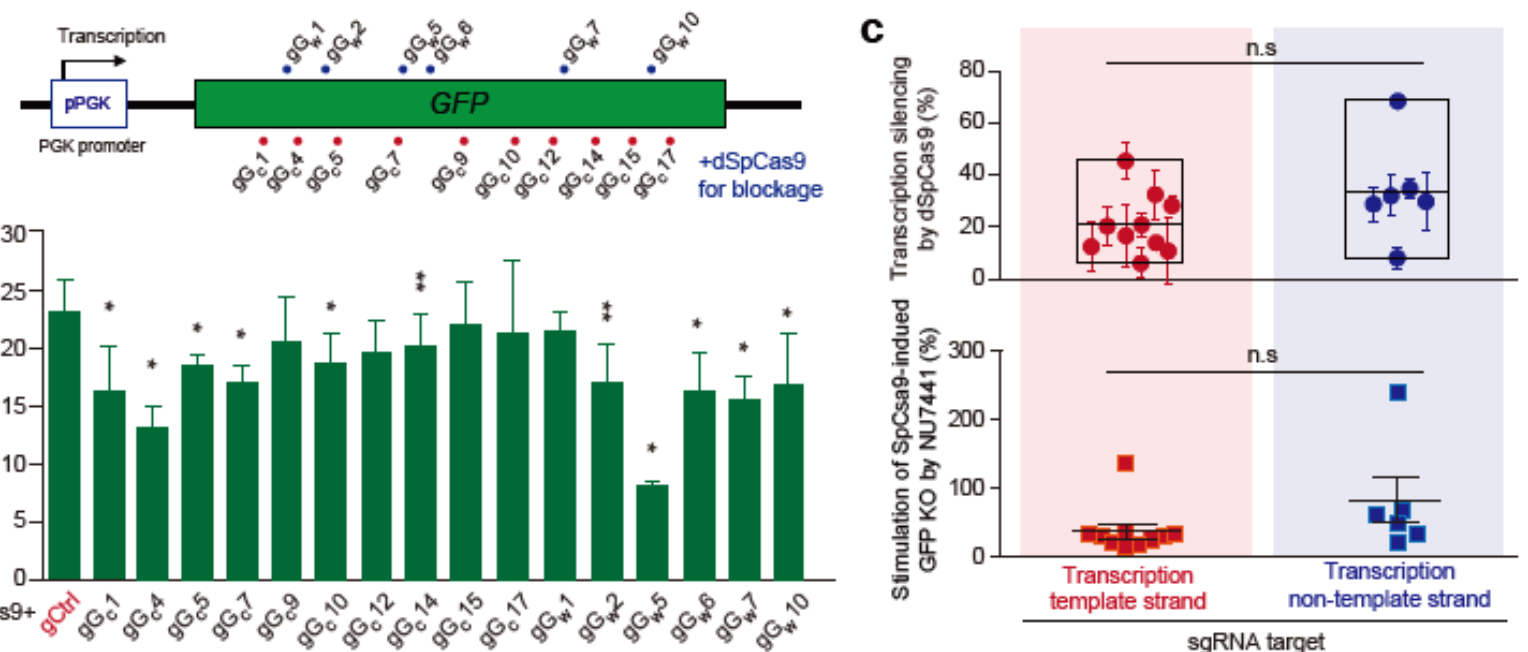

d

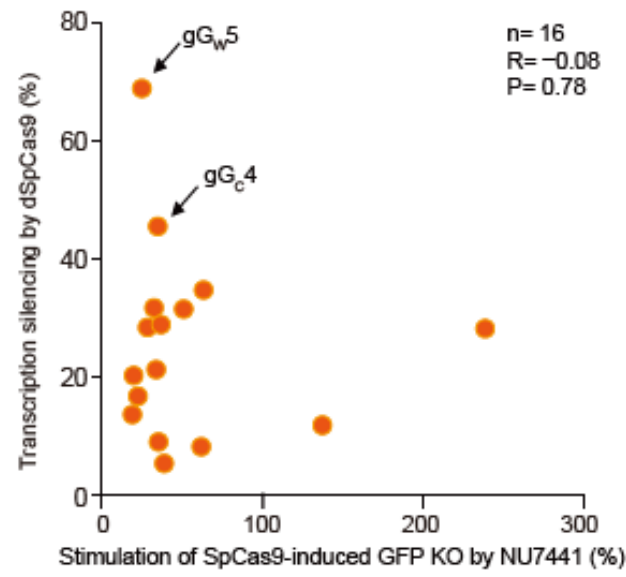

Stimulation of SpCas9-induced GFP KO by NU7441 (\%)

e
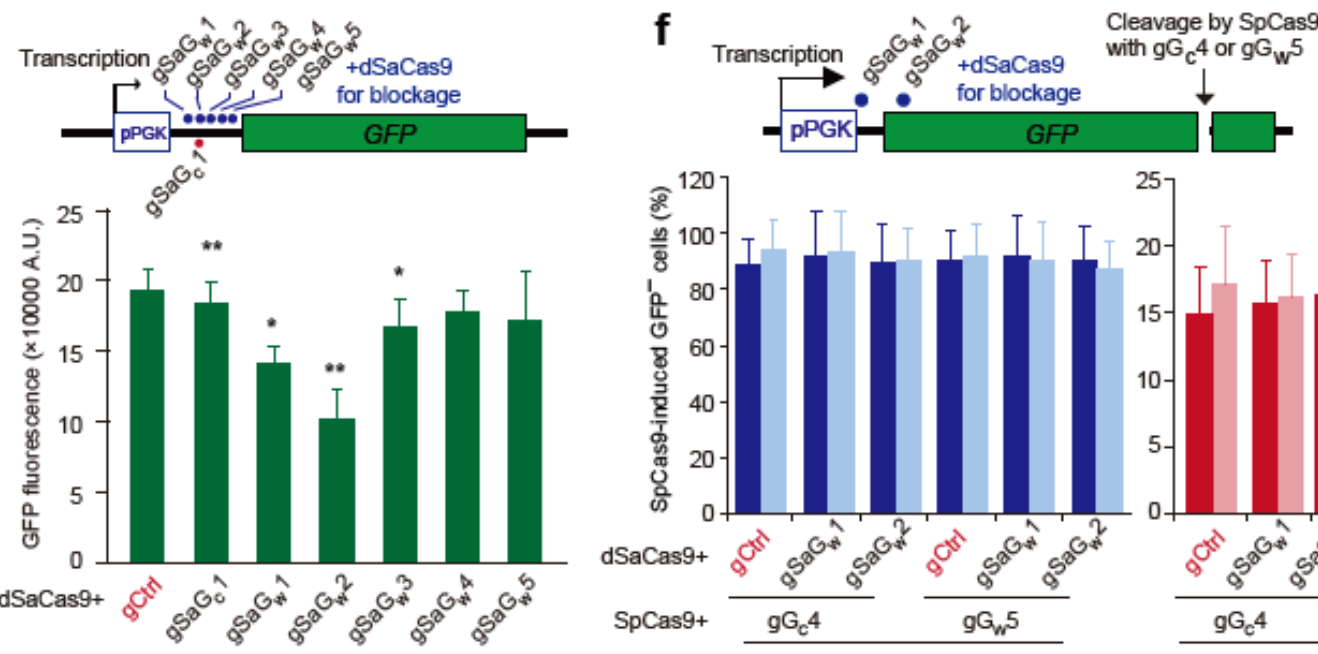

-DMSO NU7441

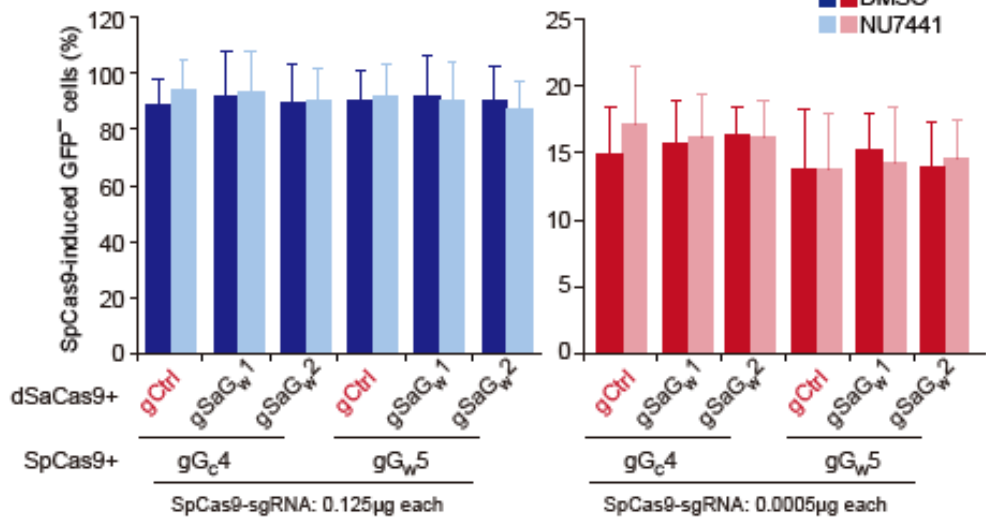

Figure 5

Transcription has no effect on c-NHEJ in repair of Cas9-induced DSBs. a dSpCas9-mediated transcriptional silencing in mESC containing PPGK-GFP expression cassette. The mean fluorescence intensity of GFP indicates relative transcription. $\mathbf{b}$ Involvement of c-NHEJ in SpCas9-induced DSBs in mESC containing pPGK-GFP expression cassette. Cells were transfected with a low amount of individual

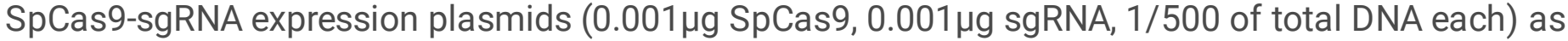


shown and treated with NU7441 at 6 h post-transfection. Relative NHEJ $(n=3)$ was calculated by normalizing DMSO treatment to 1.0. c Analysis of strand bias in transcriptional silencing (top) and cNHEJ involvement (bottom) between transcription template strand and transcription non-template strand targeted by dSpCas9-sgRNA or SpCas9-sgRNA. Transcriptional silencing and c-NHEJ involvement were defined as the percentage of GFP fluorescence intensity reduced by dSpCas9-sgRNA and the percentage of SpCas9-induced NHEJ stimulated by NU7441, respectively. $\mathbf{d}$ Correlation between dSpCas9-mediated transcriptional silencing and c-NHEJ involvement in repair of SpCas9-induced DSBs. Each circle indicates the level of dSpCas9-mediated transcriptional silencing and stimulation of SpCas9-induced NHEJ by NU7441 at the same target. Two sgRNAs $\mathrm{gG}_{\mathrm{W}} 5$ and $\mathrm{gG}_{\mathrm{C}} 4$ are indicated by arrows for their strong effect on transcriptional silencing. e dSaCas9-mediated transcriptional silencing in mESC containing $p P G K$-GFP expression cassette. The mean fluorescence intensity of GFP indicates relative transcription. Transcription blockage by dSaCas9-gSaG 1 and dSaCas9-gSaG ${ }_{W} 2$ induced significant transcription silencing. Positions of the targets by dSaCas9-sgRNAs are indicated in the reporter. $f$ Little effect of transcription blockage by dSaCas9-gSaG ${ }_{W} 1$ and dSaCas9-gSaG ${ }_{W} 2$ on DNA-PKcs involvement in SpCas9mediated GFP gene editing. GFP ${ }^{+}$cells were co-transfected with SpCas9-sgRNA (SpCas9 and sgRNA at $0.125 \mu \mathrm{g}$ and $0.0005 \mu \mathrm{g}$ respectively) and dSaCas9-sgRNA (0.125 $\mu \mathrm{g}$ each), and frequencies of SpCas9induced $G F P^{-}$cells measured by FACS at 4 d post-transfection. Each circle indicates one independent experiment, and the mean of these independent experiments is also indicated. Error bars indicate S.E.M. Two-tailed Student's paired or unpaired t-test is indicated by * for $P<0.05$, ** for $P<0.01$, *** for $P<0.001$ and n.s. for not significant. Correlation between transcription silencing and the NHEJ increase was determined by linear regression analysis. 
a
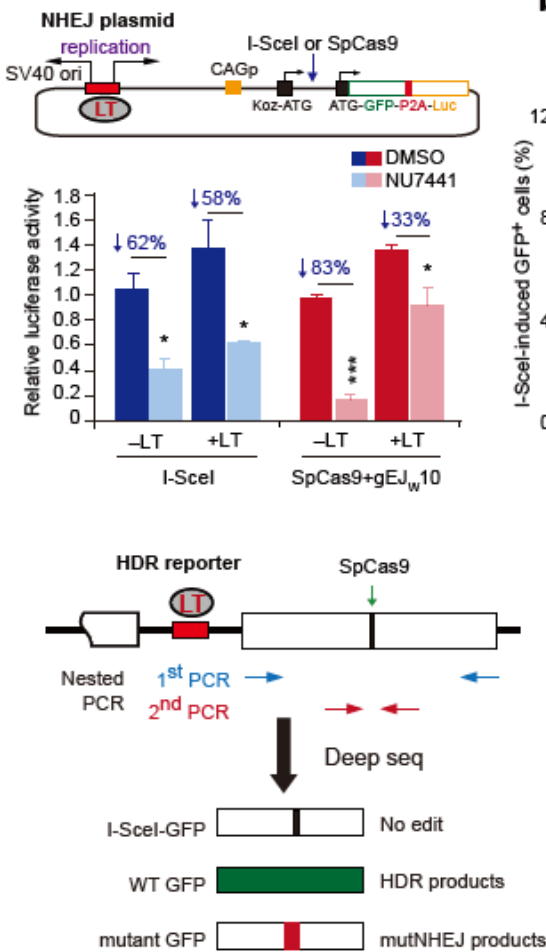

b

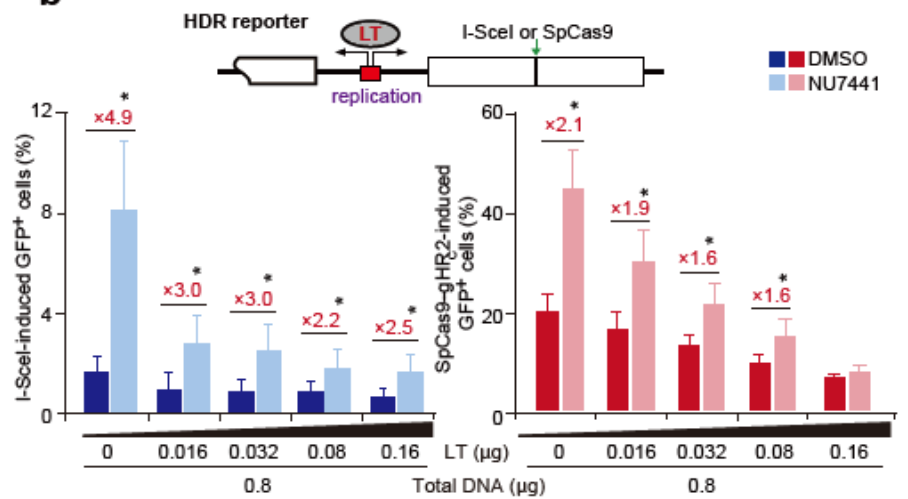

d

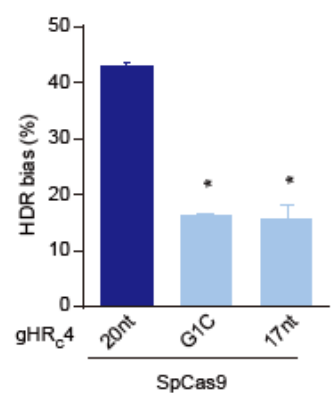

e

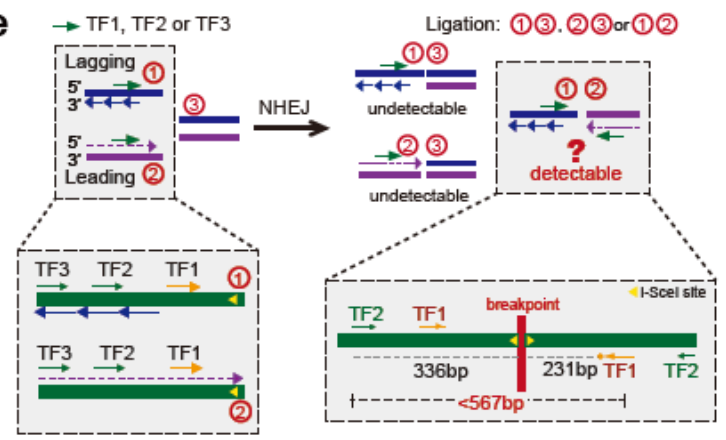

g

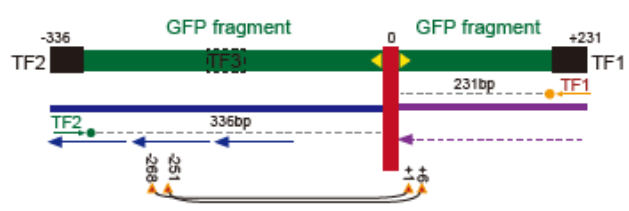

( $m E S C: n=17 ;$ U2OS: $n=23$ )

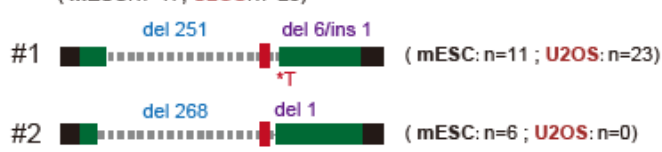

$\mathbf{f}$

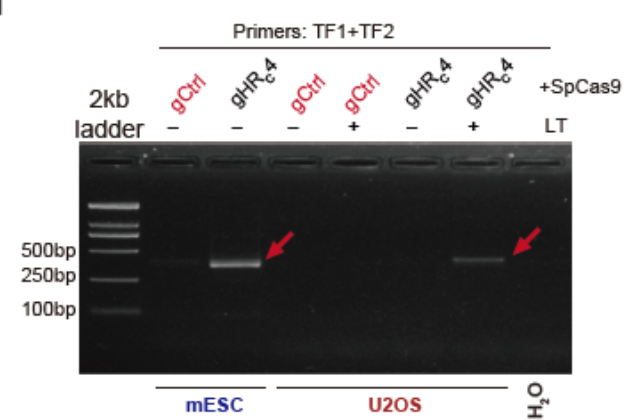

h

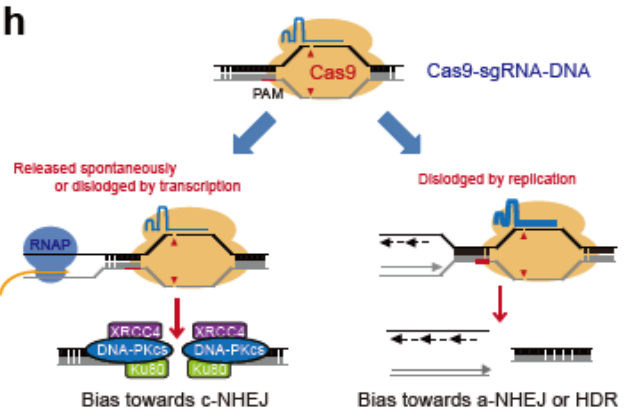

\section{Figure 6}

Replication adjacent to targets of Cas9-sgRNA suppresses c-NHEJ in repair of Cas9-induced DSBs. a Impact of local replication on DNA-PKcs involvement in NHEJ. SV40 LT can bind to the SV40 origin in a Firefly luciferase-based NHEJ reporter (Luc: Firefly luciferase; SV40 ori: SV40 DNA replication origin) to initiate replication during DNA cleavage by I-Scel or SpCas9-gEJ ${ }_{W} 10$ in 293 cells. NHEJ is represented as relative luciferase activity (i.e. ratio of Firefly luciferase activity to Renilla luciferase activity). Percentage 
of NHEJ reduction is indicated above each column. $\mathbf{b}$ Impact of local replication on DNA-PKcs involvement in HDR induced by I-Scel (left) or SpCas9-gHR 2 (right) in HDR reporter U2OS cells. SV40 LT expressed can bind to the SV40 origin in the HDR reporter to initiate replication. SV40 $L T$ was titrated as indicated. The fold of the increase is shown above each column. c Analysis schematic for SpCas9induced HDR and NHEJ at the same site of the HDR reporter. HDR bias: HDR reads/ (HDR and NHEJ reads). $\mathbf{d}$ Effect of Cas9-sgRNA target binding on HDR bias in repair of Cas9-induced DSBs in HDR reporter mESC (left) and U2OS cells (right). e Detection schematic for three ends generated by a collision between a DNA replication fork and Cas9-sgRNA at cleaved target. Three primers with different distance to the end, TF1, TF2 and TF3, were screened in pairs for PCR as indicated. $\mathbf{f}$ PCR detection of palindromic sister chromatid ligation in HDR reporter mESC and U2OS cells. Expression of SpCas9-gHR 4 , empty vector control and SV40 $L T$ is indicated. PCR was performed with the primer pair of TF1 and TF2 on gDNA. g Repair junction of sister chromatid ligation by subcloning of PCR products and Sanger sequencing. Only two types of products (\#1 and \#2) were detected with the size and position of deletion (del) and insertion (ins) as indicated. ${ }^{*} \mathrm{~T}$ : insertion of a thymidine nucleotide. $\mathbf{h}$ Impact of Cas9-sgRNA target residence on local repair pathway choice. Each circle indicates one independent experiment, each in triplicates, and the mean of at least three independent experiments is also indicated. Columns indicate the mean \pm S.E.M. Statistical significance was detected by two-tailed Student's t-test: ${ }^{*}, P<0.05 ; * \star, P<0.01$ and $* * * P<0.001$.

\section{Supplementary Files}

This is a list of supplementary files associated with this preprint. Click to download.

- Additionalfile1SupplementaryFiguresS1S7.pdf

- Additionalfile2TablesS1S3.pdf 